\title{
Equivariant Gromov-Witten Invariants of Algebraic GKM Manifolds
}

\author{
Chiu-Chu Melissa LIU $^{\dagger}$ and Artan SHESHMANI ${ }^{\ddagger}$ \\ $\dagger$ Department of Mathematics, Columbia University, \\ 2990 Broadway, New York, NY 10027, USA \\ E-mail: ccliu@math.columbia.edu \\ $\ddagger$ Harvard University, Department of Mathematics (CMSA), \\ 20 Garden Street, Cambridge, MA, 02138, USA \\ E-mail: artan@cmsa.fas.harvard.edu \\ $\S$ Aarhus University, Department of Mathematics, \\ QGM, Ny Munkegade 118, 8000 Aarhus, Denmark \\ E-mail: artan@qgm.au.dk
}

Received January 16, 2017, in final form June 21, 2017; Published online July 01, 2017 https://doi.org/10.3842/SIGMA.2017.048

\begin{abstract}
An algebraic GKM manifold is a non-singular algebraic variety equipped with an algebraic action of an algebraic torus, with only finitely many torus fixed points and finitely many 1-dimensional orbits. In this expository article, we use virtual localization to express equivariant Gromov-Witten invariants of any algebraic GKM manifold (which is not necessarily compact) in terms of Hodge integrals over moduli stacks of stable curves and the GKM graph of the GKM manifold.
\end{abstract}

Key words: Gromov-Witten theory; GKM manifold; moduli space; equivariant cohomology; localization

2010 Mathematics Subject Classification: 14C05; 14D20; 14F05; 14J30; $14 \mathrm{~N} 10$

\section{Introduction}

Gromov-Witten invariants of a projective manifold $X$ are virtual counts of parametrized algebraic curves in $X$, and can be viewed as intersection numbers on moduli spaces of stable maps $\overline{\mathcal{M}}_{g, n}(X, \beta)$ to $X$. If $X$ is equipped with an algebraic action by a torus $T$, then $T$ acts on the moduli spaces $\overline{\mathcal{M}}_{g, n}(X, \beta)$, and the Gromov-Witten invariants on $X$ can, by localization, be reduced to the intersection theory on torus fixed substack $\overline{\mathcal{M}}_{g, n}(X, \beta)^{T}$ in $\overline{\mathcal{M}}_{g, n}(X, \beta)$.

An algebraic GKM manifold, named after Goresky-Kottwitz-MacPherson, is a non-singular algebraic variety equipped with an algebraic action of $T$, such that there are finitely many 0-dimensional and 1-dimensional orbits. Examples of algebraic GKM manifolds include toric manifolds, Grassmanians, flag manifolds, etc. If $X$ is an algebraic GKM manifold then each connected component of $\overline{\mathcal{M}}_{g, n}(X, \beta)^{T}$ is, up to some quasi-finite map, a product of moduli stacks of pointed stable curves, and localization computations reduce Gromov-Witten invariants of $X$ to Hodge integrals, which are intersection numbers on the moduli stacks of pointed stable curves. This algorithm was first described by Kontsevich for genus zero Gromov-Witten invariants of $\mathbb{P}^{r}$ in 1994 [21], before the construction of virtual fundamental class and the proof of virtual localization. The moduli spaces $\overline{\mathcal{M}}_{0, n}\left(\mathbb{P}^{r}, d\right)$ of genus zero stable maps to $\mathbb{P}^{r}$ are proper smooth

This paper is a contribution to the Special Issue on Combinatorics of Moduli Spaces: Integrability, Cohomology, Quantisation, and Beyond. The full collection is available at http://www.emis.de/journals/SIGMA/modulispaces-2016.html 
DM stacks, so there exists a fundamental class $\left[\overline{\mathcal{M}}_{0, n}\left(\mathbb{P}^{r}, d\right)\right] \in H_{*}\left(\overline{\mathcal{M}}_{0, n}\left(\mathbb{P}^{r}, d\right) ; \mathbb{Q}\right)$, and one may apply the classical Atiyah-Bott localization formula [1] in this case. In [10, Section 4], T. Graber and R. Pandharipande used their virtual localization formula to derive an explicit formula for all genus Gromov-Witten invariants of $\mathbb{P}^{r}$. (See also K. Behrend [3, Section 4].) H. Spielberg derived a formula of genus zero Gromov-Witten invariants of toric manifolds in his thesis [28]. Localization computations of all genus equivariant Gromov-Witten invariants of toric manifolds can be found in [23].

The main purpose of this paper is to provide details of the virtual localization calculations of all genus equivariant Gromov-Witten invariants for general algebraic GKM manifolds described on pp. 20-21 of preprint version of $[10]^{1}$, for readers who are interested in such details. We do not assume the reader is familiar with GKM manifolds and Gromov-Witten theory: in Section 2, we define algebraic GKM manifolds and their GKM graphs, following [9, 11]; in Section 3, we give a brief review of Gromov-Witten theory. The main computations and formulae are presented in Section 4: we compute all genus equivariant descendant Gromov-Witten invariants of an arbitrary algebraic GKM manifold by virtual localization, and express the answer in terms of Hodge integrals and the GKM graph of the algebraic GKM manifold. Most of Section 4 is the straightforward generalization of the $\mathbb{P}^{r}$ case discussed in [21] (genus 0) and [10, Section 4], [3, Section 4] (higher genus); see also [13, Chapter 27].

\section{Algebraic GKM manifolds}

In this section, we review the geometry of algebraic GKM manifolds, following [9], and introduce the GKM graph associated to an algebraic GKM manifold, following [11]. The GKM graph in this paper can be non-compact since we consider algebraic GKM manifolds which are not necessarily compact. In Section 4, we will see that the GKM graph contains all the information needed for computing the Gromov-Witten invariants and the equivariant Gromov-Witten invariants of the GKM manifold.

\subsection{Basic notation}

In this paper, we work over $\mathbb{C}$.

Let $X$ be a non-singular algebraic variety of dimension $r$. We say that $X$ is an algebraic GKM manifold if it is equipped with an algebraic action of a complex algebraic torus $T=\left(\mathbb{C}^{*}\right)^{m}$ with only finitely many torus fixed points and finitely many 1-dimensional orbits.

Let $N=\operatorname{Hom}\left(\mathbb{C}^{*}, T\right) \cong \mathbb{Z}^{m}$ be the lattice of 1-parameter subgroups of $T$, and let $M=$ $\operatorname{Hom}\left(T, \mathbb{C}^{*}\right)$ be the lattice of irreducible characters of $T$. Then $M=\operatorname{Hom}(N, \mathbb{Z})$ is the dual lattice of $N$. Let $N_{\mathbb{R}}=N \otimes_{\mathbb{Z}} \mathbb{R}$ and $M_{\mathbb{R}}=M \otimes_{\mathbb{Z}} \mathbb{R}$, so that they are dual real vector spaces of dimension $m$. Let $N_{\mathbb{Q}}=N \otimes_{\mathbb{Z}} \mathbb{Q}$ and let $M_{\mathbb{Q}}=M \otimes_{\mathbb{Z}} \mathbb{Q}$. Then $M_{\mathbb{Q}}$ can be canonically identified with $H_{T}^{2}$ (point; $\left.\mathbb{Q}\right)$.

Let

$$
R_{T}:=H_{T}^{*}(\{\text { point }\} ; \mathbb{Q})=H^{*}(B T ; \mathbb{Q})=\mathbb{Q}\left[u_{1}, \ldots, u_{m}\right]
$$

be the $T$-equivariant cohomology of a point, where $u_{i} \in H_{T}^{2}(B T ; \mathbb{Q})$. Let $Q_{T}=\mathbb{Q}\left(u_{1}, \ldots, u_{m}\right)$ be the fractional field of $R_{T}$.

We make the following assumption on $X$.

\section{Assumption 2.1.}

1. The set $X^{T}$ of $T$ fixed points in $X$ is non-empty.

\footnotetext{
${ }^{1}$ Available at http://www.math.ethz.ch/ rahul/loc.ps.
} 
2. The closure of a 1-dimensional orbit is either a complex projective line $\mathbb{P}^{1}$ or a complex affine line $\mathbb{C}$.

Note that 1) and 2) hold when $X$ is a proper algebraic GKM manifold. Indeed, if $X$ is a proper algebraic GKM manifold then the closure of any 1-dimensional orbit is $\mathbb{P}^{1}$.

Example 2.2. If $X$ is a non-singular toric variety defined by a finite fan, then $X$ is an algebraic GKM manifold.

Example 2.3 (the Grassmannian $\operatorname{Gr}(k, m))$. Let $\operatorname{Gr}(k, m)$ be the set of $k$-dimensional linear subspace of $\mathbb{C}^{m}$. It is a nonsingular projective variety of dimension $k(m-k)$. Let $T=\left(\mathbb{C}^{*}\right)^{m}$ act on $\mathbb{C}^{m}$ by

$$
\left(t_{1}, \ldots, t_{m}\right) \cdot\left(z_{1}, \ldots, z_{m}\right)=\left(t_{1} z_{1}, \ldots, t_{m} z_{m}\right) .
$$

Given $t \in T$, let $\phi_{t}: \mathbb{C}^{m} \rightarrow \mathbb{C}^{m}$ be defined by $\phi_{t}(z)=t \cdot z$. Let $T$ act on $\operatorname{Gr}(k, m)$ by $t \cdot V=\phi_{t}(V)$, where $V$ is a $k$-dimensional linear subspace of $\mathbb{C}^{m}$. Given $J \subset\{1, \ldots, m\}$, let $J^{c}:=\{1, \ldots, m\} \backslash J$, and define

$$
\mathbb{C}^{J}:=\left\{\left(z_{1}, \ldots, z_{m}\right) \in \mathbb{C}^{m}: z_{i}=0 \text { if } i \in J^{c}\right\} \cong \mathbb{C}^{|J|} .
$$

Note that $\phi_{t}\left(\mathbb{C}^{J}\right)=\mathbb{C}^{J}$ for any $t \in T, J \subset\{1, \ldots, n\}$.

The torus-fixed points in $\operatorname{Gr}(k, m)$ are

$$
\operatorname{Gr}(k, m)^{T}=\left\{\mathbb{C}^{J}: J \subset\{1, \ldots, n\},|J|=k\right\} .
$$

So there are $\left(\begin{array}{c}m \\ k\end{array}\right)$ torus-fixed points in $\operatorname{Gr}(k, m)$.

Let $\mathbb{C}^{J}$ and $\mathbb{C}^{J^{\prime}}$ be distinct $T$-fixed points in $\operatorname{Gr}(k, m)$. Then $\mathbb{C}^{J} \cap \mathbb{C}^{J^{\prime}}=\mathbb{C}^{J \cap J^{\prime}}$. There is a torus-fixed line connecting $\mathbb{C}^{J}$ and $\mathbb{C}^{J^{\prime}}$ if and only of $\left|J \cap J^{\prime}\right|=k-1$. In this case, $\left|J \cup J^{\prime}\right|=k+1$. The $T$-fixed lines in $\operatorname{Gr}(k, m)$ are

$$
\left\{\ell_{I, K}: I \subset K \subset\{1, \ldots, m\},|I|=k-1,|K|=k+1\right\},
$$

where

$$
\ell_{I, K}=\left\{V \in \operatorname{Gr}(k, m): \mathbb{C}^{I} \subset V \subset \mathbb{C}^{K}\right\} \cong \mathbb{P}^{1} .
$$

Suppose that $I \subset K \subset\{1, \ldots, m\}$, and $|I|=k-1,|K|=k+1$. Then $K=I \cup\left\{j_{1}, j_{2}\right\}$, where $j_{1}, j_{2} \in I^{c}$. So there are $\left(\begin{array}{c}m \\ k-1\end{array}\right)\left(\begin{array}{c}m-k+1 \\ 2\end{array}\right)$ torus-fixed lines in $\operatorname{Gr}(k, m)$.

\subsection{GKM graph}

Let $X$ be an algebraic GKM manifold of dimension $r$, so that $T=\left(\mathbb{C}^{*}\right)^{m}$ acts algebraically on $X$.

Following [11], we define a graph $\Upsilon$ as follows. Let $V(\Upsilon)$ (resp. $E(\Upsilon)$ ) denote the set of vertices (resp. edges) in $\Upsilon$.

1. (Vertices) We assign a vertex $\sigma$ to each torus fixed point $p_{\sigma}$ in $X$.

2. (Edges) We assign an edge $\epsilon$ to each 1-dimensional $O_{\epsilon}$ in $X$. Let $\ell_{e}$ be the closure of $O_{\epsilon}$.

3. (Flags) The set of flags in the graph $\Upsilon$ is given by

$$
F(\Upsilon)=\{(\epsilon, \sigma) \in E(\Upsilon) \times V(\Upsilon): \sigma \in \epsilon\}=\left\{(\epsilon, \sigma) \in E(\Upsilon) \times V(\Upsilon): p_{\sigma} \in \ell_{\epsilon}\right\}
$$

The Assumption 2.1 can be rephrased in terms of the graph $\Upsilon$. 


\section{Assumption 2.4.}

1. $V(\Upsilon)$ is non-empty.

2. Each edge in $E(\Upsilon)$ contains at least one vertex.

Let $E(\Upsilon)_{c}=\left\{\epsilon \in E(\Upsilon): \ell_{\epsilon} \cong \mathbb{P}^{1}\right\}$ be the set of compact edges in $\Upsilon$. Note that $E(\Upsilon)_{c}=E(\Upsilon)$ if $X$ is proper.

Given a vertex $\sigma \in V(\Upsilon)$, we denote by $E_{\sigma}$ the set of edges containing $\sigma$, i.e., $E_{\sigma}:=\{\epsilon \in$ $E(\Upsilon):(\epsilon, \sigma) \in F(\Upsilon)\}$. Then $\left|E_{\sigma}\right|=r$ for all $\sigma \in V(\Upsilon)$, so $\Upsilon$ is an $r$-valent graph.

Given a flag $(\epsilon, \sigma) \in F(\Upsilon)$, let $\mathbf{w}(\epsilon, \sigma) \in M=\operatorname{Hom}\left(T, \mathbb{C}^{*}\right)$ be the weight of $T$-action on $T_{p_{\sigma}} \ell_{\epsilon}$, the tangent line to $\ell_{\epsilon}$ at the fixed point $p_{\sigma}$, namely,

$$
\mathbf{w}(\epsilon, \sigma):=c_{1}^{T}\left(T_{p_{\sigma}} \ell_{\epsilon}\right) \in H_{T}^{2}\left(p_{\sigma} ; \mathbb{Z}\right) \cong M .
$$

This gives rise to a map $\mathbf{w}: F(\Upsilon) \rightarrow M$ satisfying the following properties.

1. (GKM hypothesis) Given any $\sigma \in V(\Upsilon)$, and any two distinct edges $\epsilon, \epsilon^{\prime} \in E_{\sigma}, \mathbf{w}(\epsilon, \sigma)$ and $\mathbf{w}\left(\epsilon^{\prime}, \sigma\right)$ are linearly independent in $M_{\mathbb{R}}$.

2. Any edge $\epsilon \in E_{\sigma}$ connecting the vertices $\sigma, \sigma^{\prime} \in V(\Upsilon)$ satisfies the property that:

(a) $\mathbf{w}(\epsilon, \sigma)+\mathbf{w}\left(\epsilon, \sigma^{\prime}\right)=0$.

(b) Let $E_{\sigma}=\left\{\epsilon_{1}, \ldots, \epsilon_{r}\right\}$, where $\epsilon_{r}=\epsilon$. For any $\epsilon_{i} \in E_{\sigma}$ there exists $\epsilon_{i}^{\prime} \in E_{\sigma^{\prime}}$ and $a_{i} \in \mathbb{Z}$ such that

$$
\mathbf{w}\left(\epsilon_{i}^{\prime}, \sigma^{\prime}\right)=\mathbf{w}\left(\epsilon_{i}, \sigma\right)-a_{i} \mathbf{w}(\epsilon, \sigma) .
$$

In particular, $\epsilon_{r}^{\prime}=\epsilon_{r}=\epsilon$ and $a_{r}=2$.

Let $\epsilon$ be as in item 2 above. The normal bundle of $\ell_{\epsilon} \cong \mathbb{P}^{1}$ in $X$ is given by

$$
N_{\ell_{\epsilon} / X} \cong L_{1} \oplus \cdots \oplus L_{r-1}
$$

where $L_{i}$ is a degree $a_{i} T$-equivariant line bundle over $\ell_{\epsilon}$ such that the weights of the $T$-action on the fibers $\left(L_{i}\right)_{p_{\sigma}}$ and $\left(L_{i}\right)_{p_{\sigma^{\prime}}}$ are $\mathbf{w}\left(\epsilon_{i}, \sigma\right)$ and $\mathbf{w}\left(\epsilon_{i}^{\prime}, \sigma^{\prime}\right)$, respectively. The map w: $F(\Upsilon) \rightarrow M$ is called the axial function.

Example 2.5 $(\operatorname{Gr}(k, m))$. The GKM graph of $\operatorname{Gr}(k, m)$ is a $k(m-k)$-valent graph $\Upsilon$ such that

$$
\begin{aligned}
& V(\Upsilon)=\left\{\sigma_{J}: J \subset\{1, \ldots, n\},|J|=k\right\}, \\
& E(\Upsilon)=E(\Upsilon)_{c}=\left\{\epsilon_{I, K}: I \subset K \subset\{1, \ldots, m\},|I|=k-1,|K|=k+1\right\}, \\
& F(\Upsilon)=\left\{\left(\epsilon_{I, K}, \sigma_{J}\right) \in E(\Upsilon) \times V(\Upsilon): I \subset J \subset K\right\}, \\
& \mathbf{w}\left(\epsilon_{I K}, \sigma_{I \cup\left\{j_{1}\right\}}\right)=-\mathbf{w}\left(\epsilon_{I K}, \sigma_{I \cup\left\{j_{2}\right\}}\right)=u_{j_{2}}-u_{j_{1}}, \quad j_{1}, j_{2} \in I^{c}, \quad K=I \cup\left\{j_{1}, j_{2}\right\} .
\end{aligned}
$$

We define the 1-skeleton of $X$ to be the union of 1-dimensional orbit closures:

$$
X^{1}:=\bigcup_{\epsilon \in E(\Upsilon)} \ell_{\epsilon} .
$$

The formal completion $\hat{X}$ of $X$ along the 1-skeleton $X^{1}$ (defined on p. 194 of [12]), together with the $T$-action, can be reconstructed from the graph $\Upsilon$ and $\mathbf{w}: F(\Upsilon) \rightarrow M$. We call $(\Upsilon, \mathbf{w})$ the GKM graph of $X$ with the $T$-action. If $\rho: T^{\prime} \rightarrow T$ is a homomorphism between complex algebraic tori, then $T^{\prime}$ acts on $X$ by $t^{\prime} \cdot x=\rho\left(t^{\prime}\right) \cdot x$, where $t^{\prime} \in T^{\prime}, \rho\left(t^{\prime}\right) \in T, x \in X$. If the 0 -dimensional and 1-dimensional orbits of this $T^{\prime}$-action coincide with those of the $T$-action, then the GKM graph of $X$ with this $T^{\prime}$-action is given by $\left(\Upsilon, \rho^{*} \circ \mathbf{w}\right)$, where $\rho^{*}: M=\operatorname{Hom}\left(T, \mathbb{C}^{*}\right) \rightarrow \operatorname{Hom}\left(T^{\prime}, \mathbb{C}^{*}\right)$. 


\section{Gromov-Witten theory}

In this section, we give a brief review of the Gromov-Witten theory and the equivariant GromovWitten theory.

\subsection{Moduli space of stable curves and Hodge integrals}

An $n$-pointed, genus $g$ prestable curve is a connected algebraic curve $C$ of arithmetic genus $g$ together with $n$ ordered marked points $x_{1}, \ldots, x_{n} \in C$, where $C$ has at most nodal singularities, and $x_{1}, \ldots, x_{n}$ are distinct smooth points. An $n$-pointed, genus $g$ prestable curve $\left(C, x_{1}, \ldots, x_{n}\right)$ is stable if its automorphism group is finite, or equivalently,

$$
\operatorname{Hom}_{\mathcal{O}_{C}}\left(\Omega_{C}\left(x_{1}+\cdots+x_{n}\right), \mathcal{O}_{C}\right)=0 \text {. }
$$

Let $\overline{\mathcal{M}}_{g, n}$ be the moduli space of $n$-pointed, genus $g$ stable curves, where $n, g$ are non-negative integers. We assume that $2 g-2+n>0$, so that $\overline{\mathcal{M}}_{g, n}$ is nonempty. Then $\overline{\mathcal{M}}_{g, n}$ is a proper smooth Deligne-Mumford stack of dimension $3 g-3+n[7,17,18,19]$. The tangent space of $\overline{\mathcal{M}}_{g, n}$ at a moduli point $\left[\left(C, x_{1}, \ldots, x_{n}\right)\right] \in \overline{\mathcal{M}}_{g, n}$ is given by

$$
\operatorname{Ext}_{\mathcal{O}_{C}}^{1}\left(\Omega_{C}\left(x_{1}+\cdots+x_{n}\right), \mathcal{O}_{C}\right) .
$$

Since $\overline{\mathcal{M}}_{g, n}$ is a proper smooth Deligne-Mumford stack, there is a fundamental class

$$
\left[\overline{\mathcal{M}}_{g, n}\right] \in A_{3 g-3+n}\left(\overline{\mathcal{M}}_{g, n} ; \mathbb{Q}\right),
$$

which allows us to define

$$
\int_{\overline{\mathcal{M}}_{g, n}}: A^{*}\left(\overline{\mathcal{M}}_{g, n} ; \mathbb{Q}\right) \longrightarrow \mathbb{Q}, \quad \alpha \mapsto \operatorname{deg}\left(\alpha \cap\left[\overline{\mathcal{M}}_{g, n}\right]\right)
$$

We now introduce some classes in $A^{*}\left(\overline{\mathcal{M}}_{g, n} ; \mathbb{Q}\right)$. There is a forgetful morphism $\pi: \overline{\mathcal{M}}_{g, n+1} \rightarrow$ $\overline{\mathcal{M}}_{g, n}$ given by forgetting the $(n+1)$-th marked point (and contracting the unstable irreducible component if there is one):

$$
\left[\left(C, x_{1}, \ldots, x_{n}, x_{n+1}\right)\right] \mapsto\left[\left(C^{\text {st }}, x_{1}, \ldots, x_{n}\right)\right]
$$

where $\left(C^{\text {st }}, x_{1}, \ldots, x_{n}\right)$ is the stabilization of the prestable curve $\left(C, x_{1}, \ldots, x_{n}\right) . \pi: \overline{\mathcal{M}}_{g, n+1} \rightarrow$ $\overline{\mathcal{M}}_{g, n}$ can be identified with the universal curve over $\overline{\mathcal{M}}_{g, n}$.

- $(\lambda$ classes $)$ Let $\omega_{\pi}$ be the relative dualizing sheaf of $\pi: \overline{\mathcal{M}}_{g, n+1} \rightarrow \overline{\mathcal{M}}_{g, n}$. The Hodge bundle $\mathbb{E}=\pi_{*} \omega_{\pi}$ is a rank $g$ vector bundle over $\overline{\mathcal{M}}_{g, n}$ whose fiber over the moduli point $\left[\left(C, x_{1}, \ldots, x_{n}\right)\right] \in \overline{\mathcal{M}}_{g, n}$ is $H^{0}\left(C, \omega_{C}\right)$, the space of sections of the dualizing sheaf $\omega_{C}$ of the curve $C$. The $\lambda$ classes are defined by

$$
\lambda_{j}=c_{j}(\mathbb{E}) \in A^{j}\left(\overline{\mathcal{M}}_{g, n} ; \mathbb{Q}\right) .
$$

- $(\psi$ classes $)$ The $i$-th marked point $x_{i}$ gives rise a section $s_{i}: \overline{\mathcal{M}}_{g, n} \rightarrow \overline{\mathcal{M}}_{g, n+1}$ of the universal curve. Let $\mathbb{L}_{i}=s_{i}^{*} \omega_{\pi}$ be the line bundle over $\overline{\mathcal{M}}_{g, n}$ whose fiber over the moduli point $\left[\left(C, x_{1}, \ldots, x_{n}\right)\right] \in \overline{\mathcal{M}}_{g, n}$ is the cotangent line $T_{x_{i}}^{*} C$ of $C$ at $x_{i}$. The $\psi$ classes are defined by

$$
\psi_{i}=c_{1}\left(\mathbb{L}_{i}\right) \in A^{1}\left(\overline{\mathcal{M}}_{g, n} ; \mathbb{Q}\right) .
$$


Hodge integrals are top intersection numbers of $\lambda$ classes and $\psi$ classes:

$$
\int_{\overline{\mathcal{M}}_{g, n}} \psi_{1}^{a_{1}} \cdots \psi_{n}^{a_{n}} \lambda_{1}^{k_{1}} \cdots \lambda_{g}^{k_{g}} \in \mathbb{Q}
$$

By definition, (3.1) is zero unless

$$
a_{1}+\cdots+a_{n}+k_{1}+2 k_{2}+\cdots+g k_{g}=3 g-3+n .
$$

Using Mumford's Grothendieck-Riemann-Roch calculations in [26], Faber proved, in [8], that general Hodge integrals can be uniquely reconstructed from the $\psi$ integrals (also known as descendant integrals):

$$
\int_{\overline{\mathcal{M}}_{g, n}} \psi_{1}^{a_{1}} \cdots \psi_{n}^{a_{n}}
$$

The descendant integrals can be computed recursively by Witten's conjecture which asserts that the $\psi$ integrals (3.2) satisfy a system of differential equations known as the KdV equations [29]. The KdV equations and the string equation determine all the $\psi$ integrals (3.2) from the initial

value $\int_{\overline{\mathcal{M}}_{0,3}} 1=1$. For example, from the initial value $\int_{\overline{\mathcal{M}}_{0,3}} 1=1$ and the string equation, one can derive the following formula of genus 0 descendant integrals:

$$
\int_{\overline{\mathcal{M}}_{0, n}} \psi_{1}^{a_{1}} \cdots \psi_{n}^{a_{n}}=\frac{(n-3) !}{a_{1} ! \cdots a_{n} !},
$$

where $a_{1}+\cdots+a_{n}=n-3[21$, Section 3.3.2].

The Witten's conjecture was first proved by Kontsevich in [20]. By now, Witten's conjecture has been reproved many times (Okounkov-Pandharipande [27], Mirzakhani [24], Kim-Liu [16], Kazarian-Lando [15], Chen-Li-Liu [6], Kazarian [14], Mulase-Zhang [25], etc.).

\subsection{Moduli of stable maps}

Let $X$ be a nonsingular projective or quasi-projective variety, and let $\beta \in H_{2}(X ; \mathbb{Z})$. An $n$ pointed, genus $g$, degree $\beta$ prestable map to $X$ is a morphism $f:\left(C, x_{1}, \ldots, x_{n}\right) \rightarrow X$, where $\left(C, x_{1}, \ldots, x_{n}\right)$ is an $n$-pointed, genus $g$ prestable curve, and $f_{*}[C]=\beta$. Two prestable maps

$$
f:\left(C, x_{1}, \ldots, x_{n}\right) \rightarrow X, \quad f^{\prime}:\left(C^{\prime}, x_{1}^{\prime}, \ldots, x_{n}^{\prime}\right) \rightarrow X
$$

are isomorphic if there exists an isomorphism $\phi:\left(C, x_{1}, \ldots, x_{n}\right) \rightarrow\left(C^{\prime}, x_{1}^{\prime}, \ldots, x_{n}^{\prime}\right)$ of $n$-pointed prestable curves such that $f=f^{\prime} \circ \phi$. A prestable map $f:\left(C, x_{1}, \ldots, x_{n}\right) \rightarrow X$ is stable if its automorphism group is finite. The notion of stable maps was introduced by Kontsevich [21].

The moduli space $\overline{\mathcal{M}}_{g, n}(X, \beta)$ of $n$-pointed, genus $g$, degree $\beta$ stable maps to $X$ is a DeligneMumford stack which is proper when $X$ is projective [5].

\subsection{Obstruction theory and virtual fundamental classes}

The tangent space $T^{1}$ and the obstruction space $T^{2}$ at a moduli point $\left[f:\left(C, x_{1}, \ldots, x_{n}\right) \rightarrow\right.$ $X] \in \overline{\mathcal{M}}_{g, n}(X, \beta)$ fit in the tangent-obstruction exact sequence:

$$
\begin{aligned}
0 & \rightarrow \operatorname{Ext}_{\mathcal{O}_{C}}^{0}\left(\Omega_{C}\left(x_{1}+\cdots+x_{n}\right), \mathcal{O}_{C}\right) \rightarrow H^{0}\left(C, f^{*} T X\right) \rightarrow T^{1} \\
& \rightarrow \operatorname{Ext}_{\mathcal{O}_{C}}^{1}\left(\Omega_{C}\left(x_{1}+\cdots+x_{n}\right), \mathcal{O}_{C}\right) \rightarrow H^{1}\left(C, f^{*} T X\right) \rightarrow T^{2} \rightarrow 0,
\end{aligned}
$$

where 
- $\operatorname{Ext}_{\mathcal{O}_{C}}^{0}\left(\Omega_{C}\left(x_{1}+\cdots+x_{n}\right), \mathcal{O}_{C}\right)$ is the space of infinitesimal automorphisms of the domain $\left(C, x_{1}, \ldots, x_{n}\right)$

- $\operatorname{Ext}_{\mathcal{O}_{C}}^{1}\left(\Omega_{C}\left(x_{1}+\cdots+x_{n}\right), \mathcal{O}_{C}\right)$ is the space of infinitesimal deformations of the domain $\left(C, x_{1}, \ldots, x_{n}\right)$,

- $H^{0}\left(C, f^{*} T X\right)$ is the space of infinitesimal deformations of the map $f$, and

- $H^{1}\left(C, f^{*} T X\right)$ is the space of obstructions to deforming the map $f$.

$T^{1}$ and $T^{2}$ form sheaves $\mathcal{T}^{1}$ and $\mathcal{T}^{2}$ on the moduli space $\overline{\mathcal{M}}_{g, n}(X, \beta)$.

We say $X$ is convex if $H^{1}\left(C, f^{*} T X\right)=0$ for all genus 0 stable maps $f$. Projective spaces $\mathbb{P}^{n}$, or more generally, generalized flag varieties $G / P$, are examples of convex varieties. When $X$ is convex and $g=0$, the obstruction sheaf $\mathcal{T}^{2}=0$, and the moduli space $\overline{\mathcal{M}}_{0, n}(X, \beta)$ is a smooth Deligne-Mumford stack.

In general, $\overline{\mathcal{M}}_{g, n}(X, \beta)$ is a singular Deligne-Mumford stack equipped with a perfect obstruction theory: there is a two term complex of locally free sheaves $E \rightarrow F$ on $\overline{\mathcal{M}}_{g, n}(X, \beta)$ such that

$$
0 \rightarrow \mathcal{T}^{1} \rightarrow F^{\vee} \rightarrow E^{\vee} \rightarrow \mathcal{T}^{2} \rightarrow 0
$$

is an exact sequence of sheaves (see [4] for the complete definition of a perfect obstruction theory). The virtual dimension $d^{\text {vir }}$ of $\overline{\mathcal{M}}_{g, n}(X, \beta)$ is the rank of the virtual tangent bundle $T^{\mathrm{vir}}=F^{\vee}-E^{\vee}$,

$$
d^{\mathrm{vir}}=\int_{\beta} c_{1}(T X)+(\operatorname{dim} X-3)(1-g)+n .
$$

Suppose that $\overline{\mathcal{M}}_{g, n}(X, \beta)$ is proper. Then there is a virtual fundamental class

$$
\left[\overline{\mathcal{M}}_{g, n}(X, \beta)\right]^{\mathrm{vir}} \in A_{d^{\mathrm{vir}}}\left(\overline{\mathcal{M}}_{g, n}(X, \beta) ; \mathbb{Q}\right) .
$$

The virtual fundamental class has been constructed by Li-Tian [22], Behrend-Fantechi [4], Behrend [2] in algebraic Gromov-Witten theory. The virtual fundamental class allows us to define

$$
\int_{\left[\overline{\mathcal{M}}_{g, n}(X, \beta)\right]^{\mathrm{vir}}}: A^{*}\left(\overline{\mathcal{M}}_{g, n}(X, \beta) ; \mathbb{Q}\right) \longrightarrow \mathbb{Q}, \quad \alpha \mapsto \operatorname{deg}\left(\alpha \cap\left[\overline{\mathcal{M}}_{g, n}(X, \beta)\right]^{\mathrm{vir}}\right) .
$$

\subsection{Gromov-Witten invariants}

Let $X$ be a nonsingular projective variety. Gromov-Witten invariants are rational numbers defined by applying

$$
\int_{\left[\overline{\mathcal{M}}_{g, n}(X, \beta)\right]^{\mathrm{vir}}}: A^{*}\left(\overline{\mathcal{M}}_{g, n}(X, \beta)\right) \rightarrow \mathbb{Q}
$$

to certain classes in $A^{*}\left(\overline{\mathcal{M}}_{g, n}(X, \beta)\right)$.

Let $\mathrm{ev}_{i}: \overline{\mathcal{M}}_{g, n}(X, \beta) \rightarrow X$ be the evaluation at the $i$-th marked point: $\operatorname{ev}_{i}$ sends $\left[f:\left(C, x_{1}, \ldots\right.\right.$, $\left.\left.x_{n}\right) \rightarrow X\right] \in \overline{\mathcal{M}}_{g, n}(X, \beta)$ to $f\left(x_{i}\right) \in X$. Given $\gamma_{1}, \ldots, \gamma_{n} \in A^{*}(X)$, define

$$
\left\langle\gamma_{1}, \ldots, \gamma_{n}\right\rangle_{g, \beta}^{X}=\int_{\left[\overline{\mathcal{M}}_{g, n}(X, \beta)\right]^{\mathrm{vir}}} \operatorname{ev}_{1}^{*} \gamma_{1} \cup \cdots \cup \mathrm{ev}_{n}^{*} \gamma_{n} \in \mathbb{Q} .
$$

These are known as the primary Gromov-Witten invariants of $X$. More generally, we may also view $\left[\overline{\mathcal{M}}_{g, n}(X, \beta)\right]^{\text {vir }}$ as a class in $H_{2 d \text { vir }}\left(\overline{\mathcal{M}}_{g, n}(X, \beta)\right)$. Then (3.3) is defined for ordinary 
cohomology classes $\gamma_{1}, \ldots, \gamma_{n} \in H^{*}(X)$, including odd cohomology classes which do not come from $A^{*}\left(\overline{\mathcal{M}}_{g, n}(X, \beta)\right)$.

Let $\pi: \overline{\mathcal{M}}_{g, n+1}(X, \beta) \rightarrow \overline{\mathcal{M}}_{g, n}(X, \beta)$ be the universal curve. For $i=1, \ldots, n$, let $s_{i}: \overline{\mathcal{M}}_{g, n}(X, \beta)$ $\rightarrow \overline{\mathcal{M}}_{g, n+1}(X, \beta)$, be the section which corresponds to the $i$-th marked point. Let $\omega_{\pi} \rightarrow$ $\overline{\mathcal{M}}_{g, n+1}(X, \beta)$ be the relative dualizing sheaf of $\pi$, and let $\mathbb{L}_{i}=s_{i}^{*} \omega_{\pi}$ be the line bundle over $\overline{\mathcal{M}}_{g, n}(X, \beta)$ whose fiber at the moduli point $\left[f:\left(C, x_{1}, \ldots, x_{n}\right) \rightarrow X\right] \in \overline{\mathcal{M}}_{g, n}(X, \beta)$ is the cotangent line $T_{x_{i}}^{*} C$ at the $i$-th marked point $x_{i}$. The $\psi$-classes are defined to be

$$
\psi_{i}:=c_{1}\left(\mathbb{L}_{i}\right) \in A^{1}\left(\overline{\mathcal{M}}_{g, n}(X, \beta)\right), \quad i=1, \ldots, n .
$$

We use the same notation $\psi_{i}$ to denote the corresponding classes in the ordinary cohomology group $H^{2}\left(\overline{\mathcal{M}}_{g, n}(X, \beta)\right)$.

Genus $g$, degree $\beta$ descendant Gromov-Witten invariants of $X$ are defined by

$$
\left\langle\tau_{a_{1}}\left(\gamma_{1}\right) \cdots \tau_{a_{n}}\left(\gamma_{n}\right)\right\rangle_{g, \beta}^{X}:=\int_{\left[\overline{\mathcal{M}}_{g, n}(X, \beta)\right]^{\mathrm{vir}}} \operatorname{ev}_{1}^{*} \gamma_{1} \cup \psi_{1}^{a_{1}} \cup \cdots \cup \mathrm{ev}_{n}^{*} \gamma_{n} \cup \psi_{n}^{a_{n}} \in \mathbb{Q} .
$$

Suppose that $\gamma_{i} \in H^{d_{i}}(X)$. Then (3.4) is zero unless

$$
\sum_{i=1}^{n}\left(d_{i}+2 a_{i}-2\right)=2\left(\int_{\beta} c_{1}(T X)+(\operatorname{dim} X-3)(1-g)\right) .
$$

\subsection{Equivariant Gromov-Witten invariants}

Let $X$ be a non-singular projective or quasi-projective algebraic variety, equipped with an algebraic action of $T=\left(\mathbb{C}^{*}\right)^{m}$. Then $T$ acts on $\overline{\mathcal{M}}_{g, n}(X, \beta)$ by

$$
t \cdot\left[f:\left(C, x_{1}, \ldots, x_{n}\right) \rightarrow X\right] \mapsto\left[t \cdot f:\left(C, x_{1}, \ldots, x_{n}\right) \rightarrow X\right],
$$

where $(t \cdot f)(z)=t \cdot f(z), z \in C$. The evaluation maps ev ${ }_{i}: \overline{\mathcal{M}}_{g, n}(X, \beta) \rightarrow X$ are $T$-equivariant and induce $\mathrm{ev}_{i}^{*}: A_{T}^{*}(X ; \mathbb{Q}) \rightarrow A_{T}^{*}\left(\overline{\mathcal{M}}_{g, n}(X, \beta) ; \mathbb{Q}\right)$.

\subsubsection{Defintion when $\overline{\mathcal{M}}_{g, n}(X, \beta)$ is proper}

Suppose that $\overline{\mathcal{M}}_{g, n}(X, \beta)$ is proper, so that there are virtual fundamental classes

$$
\left[\overline{\mathcal{M}}_{g, n}(X, \beta)\right]^{\mathrm{vir}} \in A_{d^{\mathrm{vir}}}\left(\overline{\mathcal{M}}_{g, n}(X, \beta) ; \mathbb{Q}\right), \quad\left[\overline{\mathcal{M}}_{g, n}(X, \beta)\right]_{T}^{\mathrm{vir}} \in A_{d^{\mathrm{vir}}}^{T}\left(\overline{\mathcal{M}}_{g, n}(X, \beta) ; \mathbb{Q}\right),
$$

where

$$
d^{\mathrm{vir}}=\int_{\beta} c_{1}(T X)+(\operatorname{dim} X-3)(1-g)+n .
$$

Given $\gamma_{i} \in A^{d_{i}}(X ; \mathbb{Q})=H^{2 d_{i}}(X ; \mathbb{Q})$ and $a_{i} \in \mathbb{Z}_{\geq 0}$, define $\left\langle\tau_{a_{i}}\left(\gamma_{1}\right) \cdots \tau_{a_{n}}\left(\gamma_{n}\right)\right\rangle_{g, \beta}^{X}$ as in Section 3.4:

$$
\left\langle\tau_{a_{1}}\left(\gamma_{1}\right) \cdots \tau_{a_{n}}\left(\gamma_{n}\right)\right\rangle_{g, \beta}^{X}=\int_{\left[\overline{\mathcal{M}}_{g, n}(X, \beta)\right]^{\mathrm{vir}}} \prod_{i=1}^{n}\left(\mathrm{ev}_{i}^{*} \gamma_{i} \cup \psi_{i}^{a_{i}}\right) \in \mathbb{Q} .
$$

By definition, (3.5) is zero unless $\sum_{i=1}^{n}\left(d_{i}+a_{i}\right)=d^{\text {vir }}$. In this case,

$$
\left\langle\tau_{a_{1}}\left(\gamma_{1}\right) \cdots \tau_{a_{n}}\left(\gamma_{n}\right)\right\rangle_{g, \beta}^{X}=\int_{\left[\overline{\mathcal{M}}_{g, n}(X, \beta)\right]_{T}^{\mathrm{vir}}} \prod_{i=1}^{n}\left(\mathrm{ev}_{i}^{*} \gamma_{i}^{T} \cup\left(\psi_{i}^{T}\right)^{a_{i}}\right),
$$


where $\gamma_{i}^{T} \in A_{T}^{d_{i}}(X)$ is any $T$-equivariant lift of $\gamma_{i} \in A^{d_{i}}(X)$, and $\psi_{i}^{T} \in A_{T}^{1}\left(\overline{\mathcal{M}}_{g, n}(X, \beta)\right)$ is any $T$-equivariant lift of $\psi_{i} \in A^{1}\left(\overline{\mathcal{M}}_{g, n}(X, \beta)\right)$.

In this paper, we fix a choice of $\psi_{i}^{T}$ as follows. A stable map $f:\left(C, x_{1}, \ldots, x_{n}\right) \rightarrow X$ induces $\mathbb{C}$-linear maps $T_{x_{i}} C \rightarrow T_{f\left(x_{i}\right)} X$ for $i=1, \ldots, n$. This gives rise to $\mathbb{L}_{i}^{\vee} \rightarrow \operatorname{ev}_{i}^{*} T X$. The $T$-action on $X$ induces a $T$-action on $T X$, so that $T X$ is a $T$-equivariant vector bundle over $X$, and $\mathrm{ev}_{i}^{*} T X$ is a $T$-equivariant vector bundle over $\overline{\mathcal{M}}_{g, n}(X, \beta)$.

We define

$$
\psi_{i}^{T}=c_{1}^{T}\left(\mathbb{L}_{i}\right) \in A_{T}^{1}\left(\overline{\mathcal{M}}_{g, n}(X, \beta)\right), \quad i=1, \ldots, n .
$$

Then $\psi_{i}^{T}$ is a $T$-equivariant lift of $\psi_{i}=c_{1}\left(\mathbb{L}_{i}\right) \in A^{1}\left(\overline{\mathcal{M}}_{g, n}(X, \beta)\right)$.

Given $\gamma_{i}^{T} \in A_{T}^{d_{i}}(X ; \mathbb{Q})$, we define genus $g$, degree $\beta T$-equivariant descendant Gromov-Witten invariants

$$
\begin{aligned}
\left\langle\tau_{a_{1}}\left(\gamma_{1}^{T}\right), \ldots, \tau_{a_{n}}\left(\gamma_{n}^{T}\right)\right\rangle_{g, \beta}^{X_{T}}:= & \int_{\left[\overline{\mathcal{M}}_{g, n}(X, \beta)\right]_{T}^{\mathrm{vir}}} \prod_{i=1}^{n}\left(\mathrm{ev}_{i}^{*} \gamma_{i}^{T} \cup\left(\psi_{i}^{T}\right)^{a_{i}}\right) \\
& \in \mathbb{Q}\left[u_{1}, \ldots, u_{m}\right]\left(\sum_{i=1}^{n}\left(d_{i}+a_{i}\right)-d^{\mathrm{vir}}\right),
\end{aligned}
$$

where $\mathbb{Q}\left[u_{1}, \ldots, u_{m}\right](k)$ is the space of degree $k$ homogeneous polynomials in $u_{1}, \ldots, u_{m}$ with rational coefficients. In particular,

$$
\left\langle\tau_{a_{1}}\left(\gamma_{1}^{T}\right), \ldots, \tau_{a_{n}}\left(\gamma_{n}^{T}\right)\right\rangle_{g, \beta}^{X_{T}}= \begin{cases}0, & \sum_{i=1}^{n}\left(d_{i}+a_{i}\right)<d^{\mathrm{vir}}, \\ \left\langle\tau_{a_{1}}\left(\gamma_{1}\right), \ldots, \tau_{a_{n}}\left(\gamma_{n}\right)\right\rangle_{g, \beta}^{X} \in \mathbb{Q}, & \sum_{i=1}^{n}\left(d_{i}+a_{i}\right)=d^{\mathrm{vir}}\end{cases}
$$

where $\gamma_{i} \in A^{d_{i}}(X ; \mathbb{Q})$ is the image of $\gamma_{i}^{T}$ under $A_{T}^{d_{i}}(X ; \mathbb{Q}) \rightarrow A^{d_{i}}(X ; \mathbb{Q})$.

Let $\overline{\mathcal{M}}_{g, n}(X, \beta)^{T} \subset \overline{\mathcal{M}}_{g, n}(X, \beta)$ be the substack of $T$-fixed points, and let $i: \overline{\mathcal{M}}_{g, n}(X, \beta)^{T} \rightarrow$ $\overline{\mathcal{M}}_{g, n}(X, \beta)$ be the inclusion. Let $N^{\text {vir }}$ be the virtual normal bundle of substack $\overline{\mathcal{M}}_{g, n}(X, \beta)^{T}$ in $\overline{\mathcal{M}}_{g, n}(X, \beta)$; in general, $N^{\text {vir }}$ has different ranks on different connected components of $\overline{\mathcal{M}}_{g, n}(X, \beta)^{T}$. By virtual localization,

$$
\int_{\left[\overline{\mathcal{M}}_{g, n}(X, \beta)\right]_{T}^{\mathrm{vir}}} \prod_{i=1}^{n}\left(\mathrm{ev}_{i}^{*} \gamma_{i}^{T} \cup\left(\psi_{i}^{T}\right)^{a_{i}}\right)=\int_{\left[\overline{\mathcal{M}}_{g, n}(X, \beta)^{T}\right]_{T}^{\mathrm{vir}}} \frac{i^{*} \prod_{i=1}^{n}\left(\mathrm{ev}_{i}^{*} \gamma_{i}^{T} \cup\left(\psi_{i}^{T}\right)^{a_{i}}\right)}{e^{T}\left(N^{\mathrm{vir}}\right)} .
$$

\subsubsection{Definition when $\overline{\mathcal{M}}_{g, n}(X, \beta)$ is not proper}

Suppose that $\overline{\mathcal{M}}_{g, n}(X, \beta)$ is not proper, but $\overline{\mathcal{M}}_{g, n}(X, \beta)^{T}$ is. Then the left hand side of (3.6) is not defined, but the right hand side of (3.6) is. In this case, we use the right hand side of (3.6) to define $T$-equivariant Gromov-Witten invariants:

$$
\left\langle\tau_{a_{1}}\left(\gamma_{1}^{T}\right), \ldots, \tau_{a_{n}}\left(\gamma_{n}^{T}\right)\right\rangle_{g, \beta}^{X_{T}}:=\int_{\left[\overline{\mathcal{M}}_{g, n}(X, \beta)^{T}\right]_{T}^{\mathrm{vir}}} \frac{i^{*} \prod_{i=1}^{n}\left(\mathrm{ev}_{i}^{*} \gamma_{i}^{T} \cup\left(\psi_{i}^{T}\right)^{a_{i}}\right)}{e^{T}\left(N^{\mathrm{vir}}\right)} \in Q_{T} .
$$

When $\overline{\mathcal{M}}_{g, n}(X, \beta)$ is not proper, the right hand side of (3.7) is a rational function (instead of a polynomial) in $u_{1}, \ldots, u_{m}$. It can be nonzero when $\sum_{i=1}^{n}\left(d_{i}+a_{i}\right)<d^{\text {vir }}$, and does not have a nonequivariant limit (obtained by setting $u_{i}=0$ ) in general. 


\section{Virtual Localization}

In this section, we compute all genus equivariant descendant Gromov-Witten invariants of any algebraic GKM manifold by virtual localization. This generalizes the toric case in [23, Section 5].

Let $X$ be an algebraic GKM manifold of dimension $r$, with an algebraic action of $T=\left(\mathbb{C}^{*}\right)^{m}$, and let $\Upsilon$ be the corresponding GKM graph.

\subsection{Torus fixed points and graph notation}

In this subsection, we describe the $T$-fixed points in $\overline{\mathcal{M}}_{g, n}(X, \beta)$. Following Kontsevich [21], given a stable map $f:\left(C, x_{1}, \ldots, x_{n}\right) \rightarrow X$ such that

$$
\left[f:\left(C, x_{1}, \ldots, x_{n}\right) \rightarrow X\right] \in \overline{\mathcal{M}}_{g, n}(X, \beta)^{T},
$$

we will associate a decorated graph $\vec{\Gamma}$.

We first give a formal definition.

Definition 4.1. A decorated graph $\vec{\Gamma}=(\Gamma, \vec{f}, \vec{d}, \vec{g}, \vec{s})$ for $n$-pointed, genus $g$, degree $\beta$ stable maps to $X$ consists of the following data.

1. $\Gamma$ is a compact, connected 1-dimensional $\mathrm{CW}$ complex. We denote the set of vertices (resp. edges) in $\Gamma$ by $V(\Gamma)$ (resp. $E(\Gamma)$ ). Let

$$
F(\Gamma)=\{(e, v) \in E(\Gamma) \times V(\Gamma) \mid v \in e\}
$$

be the set of flags in $\Gamma$.

2. The label map $\vec{f}: V(\Gamma) \cup E(\Gamma) \rightarrow V(\Upsilon) \cup E(\Upsilon)_{c}$ sends a vertex $v \in V(\Gamma)$ to a vertex $\sigma_{v} \in V(\Upsilon)$, and sends an edge $e \in E(\Gamma)$ to an edge $\epsilon_{e} \in E(\Upsilon)_{c}$. Moreover, $\vec{f}$ defines a map from the graph $\Gamma$ to the graph $\Upsilon$ : if $(e, v)$ is a flag in $\Gamma$ then $\left(\epsilon_{e}, \sigma_{v}\right)$ is a flag in $\Upsilon$.

3. The degree map $\vec{d}: E(\Gamma) \rightarrow \mathbb{Z}_{>0}$ sends an edge $e \in E(\Gamma)$ to a positive integer $d_{e}$.

4. The genus map $\vec{g}: V(\Gamma) \rightarrow \mathbb{Z}_{\geq 0}$ sends a vertex $v \in V(\Gamma)$ to a non-negative integer $g_{v}$.

5. The marking map $\vec{s}:\{1,2, \ldots, n\} \rightarrow V(\Gamma)$ is defined if $n>0$.

The above maps satisfy the following two constraints:

(i) (topology of the domain) $\sum_{v \in V(\Gamma)} g_{v}+|E(\Gamma)|-|V(\Gamma)|+1=g$.

(ii) (topology of the map) $\sum_{e \in E(\Gamma)} d_{e}\left[\ell_{\epsilon_{e}}\right]=\beta$.

Let $G_{g, n}(X, \beta)$ be the set of all decorated graphs $\vec{\Gamma}=(\Gamma, \vec{f}, \vec{d}, \vec{g}, \vec{s})$ satisfying the above constraints.

We now describe the geometry and combinatorics of a stable map $f:\left(C, x_{1}, \ldots, x_{n}\right) \rightarrow X$ which represents a $T$-fixed point in $\overline{\mathcal{M}}_{g, n}(X, \beta)$.

For any $t \in T$, there exists an automorphism $\phi_{t}$ of $\left(C, x_{1}, \ldots, x_{n}\right)$ such that $t \cdot f(z)=f \circ \phi_{t}(z)$ for any $z \in C$. Let $C^{\prime}$ be an irreducible component of $C$, and let $f^{\prime}=\left.f\right|_{C^{\prime}}: C^{\prime} \rightarrow X$. There are two possibilities:

Case 1: $f^{\prime}$ is a constant map, and $f\left(C^{\prime}\right)=\left\{p_{\sigma}\right\}$, where $p_{\sigma}$ is a fixed point in $X$ associated to some $\sigma \in V(\Upsilon)$.

Case 2: $C^{\prime} \cong \mathbb{P}^{1}$ and $f\left(C^{\prime}\right)=\ell_{\epsilon}$, where $\ell_{\epsilon}$ is a $T$-invariant $\mathbb{P}^{1}$ in $X$ associated to some $\epsilon \in E(\Upsilon)_{c}$. 
We define a decorated graph $\vec{\Gamma}$ associated to $f:\left(C, x_{1}, \ldots, x_{n}\right) \rightarrow X$ as follows.

1. (Vertices) We assign a vertex $v$ to each connected component $C_{v}$ of $f^{-1}\left(X^{T}\right)$.

(a) (label) $f\left(C_{v}\right)=\left\{p_{\sigma}\right\}$ for some $\sigma \in V(\Upsilon)$; we define $\vec{f}(v)=\sigma_{v}=\sigma$.

(b) (genus) $C_{v}$ is a curve or a point. If $C_{v}$ is a curve then we define $\vec{g}(v)=g_{v}$ to be the arithmetic genus of $C_{v}$; if $C_{v}$ is a point then we define $\vec{g}(v)=g_{v}=0$.

(c) (marking) For $i=1, \ldots, n$, define $\vec{s}(i)=v$ if $x_{i} \in C_{v}$.

2. (Edges) For any $\epsilon \in E(\Upsilon)$, let $O_{\epsilon} \cong \mathbb{C}^{*}$ be the 1-dimensional orbit whose closure is $\ell_{\epsilon}$. Then

$$
X^{1} \backslash X^{T}=\bigsqcup_{\epsilon \in E(\Upsilon)} O_{\epsilon}
$$

where the right hand side is a disjoint union of connected components. We assign an edge $e$ to each connected component $O_{e} \cong \mathbb{C}^{*}$ of $f^{-1}\left(X^{1} \backslash X^{T}\right)$.

(a) (label) Let $C_{e} \cong \mathbb{P}^{1}$ be the closure of $O_{e}$. Then $f\left(C_{e}\right)=\ell_{\epsilon}$ for some $\epsilon \in E(\Upsilon)_{c}$; we define $\vec{f}(e)=\epsilon_{e}=\epsilon$.

(b) (degree) We define $\vec{d}(e)=d_{e}$ to be the degree of the map $\left.f\right|_{C_{e}}: C_{e} \cong \mathbb{P}^{1} \rightarrow \ell_{\epsilon} \cong \mathbb{P}^{1}$.

3. (Flags) The set of flags in the graph $\Gamma$ is defined by

$$
F(\Gamma)=\left\{(e, v) \in E(\Gamma) \times V(\Gamma) \mid C_{e} \cap C_{v} \neq \varnothing\right\} .
$$

The above 1), 2), 3) define a decorated graph $\vec{\Gamma}=(\Gamma, \vec{f}, \vec{d}, \vec{g}, \vec{s})$ satisfying the constraints (i) and (ii) in Definition 4.1. Therefore $\vec{\Gamma} \in G_{g, n}(X, \beta)$. This gives a map from $\overline{\mathcal{M}}_{g, n}(X, \beta)^{T}$ to the discrete set $G_{g, n}(X, \beta)$. Let $\mathcal{F}_{\vec{\Gamma}} \subset \overline{\mathcal{M}}_{g, n}(X, \beta)^{T}$ denote the preimage of $\vec{\Gamma}$. Then

$$
\overline{\mathcal{M}}_{g, n}(X, \beta)^{T}=\bigsqcup_{\vec{\Gamma} \in G_{g, n}(X, \beta)} \mathcal{F}_{\vec{\Gamma}},
$$

where the right hand side is a disjoint union of connected components. We next describe the fixed locus $\mathcal{F}_{\vec{\Gamma}}$ associated to each decorated graph $\vec{\Gamma} \in G_{g, n}(X, \beta)$. For later convenience, we introduce some definitions.

Definition 4.2. Given a vertex $v \in V(\Gamma)$, we define

$$
E_{v}=\{e \in E(\Gamma) \mid(e, v) \in F(\Gamma)\}
$$

the set of edges emanating from $v$, and define $S_{v}=\vec{s}^{-1}(v) \subset\{1, \ldots, n\}$. The valency of $v$ is given by $\operatorname{val}(v)=\left|E_{v}\right|$. Let $n_{v}=\left|S_{v}\right|$ be the number of marked points contained in $C_{v}$. We say a vertex is stable if $2 g_{v}-2+\operatorname{val}(v)+n_{v}>0$. Let $V^{S}(\Gamma)$ be the set of stable vertices in $V(\Gamma)$. There are three types of unstable vertices:

$$
\begin{aligned}
& V^{1}(\Gamma)=\left\{v \in V(\Gamma) \mid g_{v}=0, \operatorname{val}(v)=1, n_{v}=0\right\} \\
& V^{1,1}(\Gamma)=\left\{v \in V(\Gamma) \mid g_{v}=0, \operatorname{val}(v)=n_{v}=1\right\} \\
& V^{2}(\Gamma)=\left\{v \in V(\Gamma) \mid g_{v}=0, \operatorname{val}(v)=2, n_{v}=0\right\}
\end{aligned}
$$

Then $V(\Gamma)$ is the disjoint union of $V^{1}(\Gamma), V^{1,1}(\Gamma), V^{2}(\Gamma)$, and $V^{S}(\Gamma)$.

The set of stable flags is defined to be

$$
F^{S}(\Gamma)=\left\{(e, v) \in F(\Gamma) \mid v \in V^{S}(\Gamma)\right\} .
$$


Given a decorated graph $\vec{\Gamma}=(\Gamma, \vec{f}, \vec{d}, \vec{g}, \vec{s})$, the curves $C_{e}$ and the maps $\left.f\right|_{C_{e}}: C_{e} \rightarrow \ell_{\epsilon_{e}} \subset X$ are determined by $\vec{\Gamma}$. If $v \notin V^{S}(\Gamma)$ then $C_{v}$ is a point. If $v \in V^{S}(\Gamma)$ then $C_{v}$ is a curve, and $y(e, v):=C_{e} \cap C_{v}$ is a node of $C$ for $e \in E_{v}$,

$$
\left(C_{v},\left\{y(e, v): e \in E_{v}\right\} \cup\left\{x_{i} \mid i \in S_{v}\right\}\right)
$$

is a $\left(\operatorname{val}(v)+n_{v}\right)$-pointed, genus $g_{v}$ curve, which represents a point in $\overline{\mathcal{M}}_{g_{v}, \operatorname{val}(v)+n_{v}}$. We call this moduli space $\overline{\mathcal{M}}_{g_{v}, E_{v} \cup S_{v}}$ instead of $\overline{\mathcal{M}}_{g_{v}, \operatorname{val}(v)+n_{v}}$ because we would like to label the marked points on $C_{v}$ by $E_{v} \cup S_{v}$ instead of $\left\{1,2, \ldots, \operatorname{val}(v)+n_{v}\right\}$. Then

$$
\mathcal{M}_{\vec{\Gamma}}=\prod_{v \in V^{S}(\Gamma)} \overline{\mathcal{M}}_{g_{v}, E_{v} \cup S_{v}} .
$$

The automorphism group $A_{\vec{\Gamma}}$ for any point $\left[f:\left(C, x_{1}, \ldots, x_{n}\right) \rightarrow X\right] \in \mathcal{F}_{\vec{\Gamma}}$ fits in the following short exact sequence of groups:

$$
1 \rightarrow \prod_{e \in E(\Gamma)} \mathbb{Z}_{d_{e}} \rightarrow A_{\vec{\Gamma}} \rightarrow \operatorname{Aut}(\vec{\Gamma}) \rightarrow 1
$$

where $\mathbb{Z}_{d_{e}}$ is the automorphism group of the degree $d_{e}$ morphism

$$
\left.f\right|_{C_{e}}: C_{e} \cong \mathbb{P}^{1} \rightarrow \ell_{\epsilon_{e}} \cong \mathbb{P}^{1}
$$

and $\operatorname{Aut}(\vec{\Gamma})$ is the automorphism group of the decorated graph $\vec{\Gamma}=(\Gamma, \vec{f}, \vec{d}, \vec{g}, \vec{s})$. There is a morphism $i_{\vec{\Gamma}}: \mathcal{M}_{\vec{\Gamma}} \rightarrow \overline{\mathcal{M}}_{g, n}(X, \beta)$ whose image is the fixed locus $\mathcal{F}_{\vec{\Gamma}}$ associated to $\vec{\Gamma} \in G_{g, n}(X, \beta)$. The morphism $i_{\vec{\Gamma}}$ induces an isomorphism $\left[\mathcal{M}_{\vec{\Gamma}} / A_{\vec{\Gamma}}\right] \cong \mathcal{F}_{\vec{\Gamma}}$.

\subsection{Virtual tangent and normal bundles}

Given a decorated graph $\vec{\Gamma} \in G_{g, n}(X, \beta)$ and a stable map $f:\left(C, x_{1}, \ldots, x_{n}\right) \rightarrow X$ which represents a point in the fixed locus $\mathcal{F}_{\vec{\Gamma}}$ associated to $\vec{\Gamma}$, let

$$
\begin{array}{ll}
B_{1}=\operatorname{Hom}\left(\Omega_{C}\left(x_{1}+\cdots+x_{n}\right), \mathcal{O}_{C}\right), & B_{2}=H^{0}\left(C, f^{*} T X\right), \\
B_{4}=\operatorname{Ext}^{1}\left(\Omega_{C}\left(x_{1}+\cdots+x_{n}\right), \mathcal{O}_{C}\right), & B_{5}=H^{1}\left(C, f^{*} T X\right) .
\end{array}
$$

Then $B_{1}, B_{2}, B_{4}, B_{5}$ are representations of the torus $T$, so there is a direct sum decomposition $B_{i}=B_{i}^{f} \oplus B_{i}^{m}$, where $B_{i}^{f} \subset B_{i}$ is the $T$-invariant subspace. We have the following exact sequences:

$$
\begin{aligned}
& 0 \rightarrow B_{1}^{f} \rightarrow B_{2}^{f} \rightarrow T^{1, f} \rightarrow B_{4}^{f} \rightarrow B_{5}^{f} \rightarrow T^{2, f} \rightarrow 0 \\
& 0 \rightarrow B_{1}^{m} \rightarrow B_{2}^{m} \rightarrow T^{1, m} \rightarrow B_{4}^{m} \rightarrow B_{5}^{m} \rightarrow T^{2, m} \rightarrow 0 .
\end{aligned}
$$

The irreducible components of $C$ are

$$
\left\{C_{v} \mid v \in V^{S}(\Gamma)\right\} \cup\left\{C_{e} \mid e \in E(\Gamma)\right\}
$$

The nodes of $C$ are

$$
\left\{y_{v}=C_{v} \mid v \in V^{2}(\Gamma)\right\} \cup\left\{y(e, v) \mid(e, v) \in F^{S}(\Gamma)\right\}
$$




\subsubsection{Automorphisms of the domain}

Given any $(e, v) \in F(\Gamma)$, let $y(e, v)=C_{e} \cap C_{v}$, and define

$$
w_{(e, v)}:=e^{T}\left(T_{y(e, v)} C_{e}\right)=\frac{\mathbf{w}\left(\epsilon_{e}, \sigma_{v}\right)}{d_{e}} \in H_{T}^{2}(y(e, v) ; \mathbb{Q})=M \otimes_{\mathbb{Z}} \mathbb{Q} .
$$

We have

$$
\begin{aligned}
B_{1}^{f} & =\bigoplus_{\substack{e \in E(\Gamma) \\
(e, v),\left(e, v^{\prime}\right) \in F(\Gamma)}} \operatorname{Hom}\left(\Omega_{C_{e}}\left(y(e, v)+y\left(e, v^{\prime}\right)\right), \mathcal{O}_{C_{e}}\right) \\
& =\bigoplus_{\substack{e \in E(\Gamma) \\
(e, v),\left(e, v^{\prime}\right) \in F(\Gamma)}} H^{0}\left(C_{e}, T C_{e}\left(-y(e, v)-y\left(e, v^{\prime}\right)\right),\right. \\
B_{1}^{m} & =\bigoplus_{v \in V^{1}(\Gamma),(e, v) \in F(\Gamma)} T_{y(e, v)} C_{e} .
\end{aligned}
$$

\subsubsection{Deformations of the domain}

Given any $v \in V^{S}(\Gamma)$, define a divisor $\mathbf{x}_{v}$ of $C_{v}$ by

$$
\mathbf{x}_{v}=\sum_{i \in S_{v}} x_{i}+\sum_{e \in E_{v}} y(e, v)
$$

Let $\mathbb{L}_{(e, v)}$ be the line bundle over $\overline{\mathcal{M}}_{g_{v}, E_{v} \cup S_{v}}$ whose fiber at the moduli point $\left[C_{v}, \mathbf{x}_{v}\right]$ is the cotangent line $T_{y(e, v)}^{*} C_{v}$. Let

$$
\psi_{(e, v)}=c_{1}\left(\mathbb{L}_{(e, v)}\right) \in A^{1}\left(\overline{\mathcal{M}}_{g_{v}, E_{v} \cup S_{v}}\right) .
$$

The torus $T$ acts trivially on $\overline{\mathcal{M}}_{g_{v}, E_{v} \cup S_{v}}$ and $\mathbb{L}_{(e, v)}$, so $\psi_{(e, v)}$ can also be viewed as the $T$ equivariant first Chern class of $\mathbb{L}_{(e, v)}$.

We have

$$
\begin{aligned}
& B_{4}^{f}=\bigoplus_{v \in V^{S}(\Gamma)} \operatorname{Ext}^{1}\left(\Omega_{C_{v}}\left(\mathbf{x}_{v}\right), \mathcal{O}_{C}\right)=\bigoplus_{v \in V^{S}(\Gamma)} T_{\left(C_{v}, \mathbf{x}_{v}\right)} \overline{\mathcal{M}}_{g_{v}, E_{v} \cup S_{v}}, \\
& B_{4}^{m}=\bigoplus_{v \in V^{2}(\Gamma), E_{v}=\left\{e, e^{\prime}\right\}} T_{y_{v}} C_{e} \otimes T_{y_{v}} C_{e^{\prime}} \oplus \bigoplus_{(e, v) \in F^{S}(\Gamma)} T_{y(e, v)} C_{v} \otimes T_{y(e, v)} C_{e},
\end{aligned}
$$

where

$$
\begin{aligned}
& e^{T}\left(T_{y_{v}} C_{e} \otimes T_{y_{v}} C_{e^{\prime}}\right)=w_{(e, v)}+w_{\left(e^{\prime}, v\right)}, \quad v \in V^{2}(\Gamma), \\
& e^{T}\left(T_{y(e, v)} C_{v} \otimes T_{y(e, v)} C_{e}\right)=w_{(e, v)}-\psi_{(e, v)}, \quad v \in V^{S}(\Gamma) .
\end{aligned}
$$

\subsubsection{Unifying stable and unstable vertices}

From the discussion in Sections 4.2.1 and 4.2.2,

$$
\begin{aligned}
\frac{e^{T}\left(B_{1}^{m}\right)}{e^{T}\left(B_{4}^{m}\right)}= & \prod_{v \in V^{1}(\Gamma),(e, v) \in F(\Gamma)} w_{(e, v)} \prod_{v \in V^{2}(\Gamma), E_{v}=\left\{e, e^{\prime}\right\}} \frac{1}{w_{(e, v)}+w_{\left(e^{\prime}, v\right)}} \\
& \times \prod_{v \in V^{S}(\Gamma)} \frac{1}{\prod_{e \in E_{v}}\left(w_{(e, v)}-\psi_{(e, v)}\right)} .
\end{aligned}
$$


Recall that

$$
\mathcal{M}_{\vec{\Gamma}}=\prod_{v \in V^{S}(\Gamma)} \overline{\mathcal{M}}_{g_{v}, E_{v} \cup S_{v}}
$$

To unify the stable and unstable vertices, we use the following convention for the empty sets $\overline{\mathcal{M}}_{0,1}$ and $\overline{\mathcal{M}}_{0,2}$. Let $w_{1}, w_{2}$ be formal variables.

(i) $\overline{\mathcal{M}}_{0,1}$ is a -2-dimensional space, and

$$
\int_{\overline{\mathcal{M}}_{0,1}} \frac{1}{w_{1}-\psi_{1}}=w_{1}
$$

(ii) $\overline{\mathcal{M}}_{0,2}$ is a -1-dimensional space, and

$$
\begin{aligned}
& \int_{\overline{\mathcal{M}}_{0,2}} \frac{1}{\left(w_{1}-\psi_{1}\right)\left(w_{2}-\psi_{2}\right)}=\frac{1}{w_{1}+w_{2}}, \\
& \int_{\overline{\mathcal{M}}_{0,2}} \frac{1}{w_{1}-\psi_{1}}=1 .
\end{aligned}
$$

(iii) $\mathcal{M}_{\vec{\Gamma}}=\prod_{v \in V(\Gamma)} \overline{\mathcal{M}}_{g_{v}, E_{v} \cup S_{v}}$.

With the above conventions (i), (ii), (iii), we may rewrite (4.1) as

$$
\frac{e^{T}\left(B_{1}^{m}\right)}{e^{T}\left(B_{4}^{m}\right)}=\prod_{v \in V(\Gamma)} \frac{1}{\prod_{e \in E_{v}}\left(w_{(e, v)}-\psi_{(e, v)}\right)} .
$$

The following lemma shows that the conventions (i) and (ii) are consistent with the stable case $\overline{\mathcal{M}}_{0, n}, n \geq 3$.

Lemma 4.3. For any positive integer $n$ and formal variables $w_{1}, \ldots, w_{n}$, we have

$$
\begin{aligned}
& \text { (a) } \int_{\overline{\mathcal{M}}_{0, n}} \frac{1}{\prod_{i=1}^{n}\left(w_{i}-\psi_{i}\right)}=\frac{1}{w_{1} \cdots w_{n}}\left(\frac{1}{w_{1}}+\cdots \frac{1}{w_{n}}\right)^{n-3}, \\
& \text { (b) } \int_{\overline{\mathcal{M}}_{0, n}} \frac{1}{w_{1}-\psi_{1}}=w_{1}^{2-n} .
\end{aligned}
$$

Proof. (a) The cases $n=1$ and $n=2$ follow from the definitions (4.2) and (4.3), respectively. For $n \geq 3$, we have

$$
\begin{aligned}
\int_{\overline{\mathcal{M}}_{0, n}} \frac{1}{\prod_{i=1}^{n}\left(w_{i}-\psi_{i}\right)} & =\frac{1}{w_{1} \cdots w_{n}} \int_{\overline{\mathcal{M}}_{0, n}} \frac{1}{\prod_{i=1}^{n}\left(1-\frac{\psi_{i}}{w_{i}}\right)} \\
& =\frac{1}{w_{1} \cdots w_{n}} \sum_{a_{1}+\cdots+a_{n}=n-3} w_{1}^{-a_{1}} \cdots w_{n}^{-a_{n}} \int_{\overline{\mathcal{M}}_{0, n}} \psi_{1}^{a_{1}} \cdots \psi_{n}^{a_{n}},
\end{aligned}
$$

where

$$
\int_{\overline{\mathcal{M}}_{0, n}} \psi_{1}^{a_{1}} \cdots \psi_{n}^{a_{n}}=\frac{(n-3) !}{a_{1} ! \cdots a_{n} !} .
$$


So

$$
\int_{\overline{\mathcal{M}}_{0, n}} \frac{1}{\prod_{i=1}^{n}\left(w_{i}-\psi_{i}\right)}=\frac{1}{w_{1} \cdots w_{n}}\left(\frac{1}{w_{1}}+\cdots \frac{1}{w_{n}}\right)^{n-3} .
$$

(b) The cases $n=1$ and $n=2$ follow from the definitions (4.2) and (4.4), respectively. For $n \geq 3$, we have

$$
\int_{\overline{\mathcal{M}}_{0, n}} \frac{1}{w_{1}-\psi_{1}}=\frac{1}{w_{1}} \int_{\overline{\mathcal{M}}_{0, n}} \frac{1}{1-\frac{\psi_{1}}{w_{1}}}=\frac{1}{w_{1}} w_{1}^{3-n}=w_{1}^{2-n} .
$$

\subsubsection{Deformation of the map}

Consider the normalization sequence

$$
0 \rightarrow \mathcal{O}_{C} \rightarrow \bigoplus_{v \in V^{S}(\Gamma)} \mathcal{O}_{C_{v}} \oplus \bigoplus_{e \in E(\Gamma)} \mathcal{O}_{C_{e}} \rightarrow \bigoplus_{v \in V^{2}(\Gamma)} \mathcal{O}_{y_{v}} \oplus \bigoplus_{(e, v) \in F^{S}(\Gamma)} \mathcal{O}_{y(e, v)} \rightarrow 0
$$

We twist the above short exact sequence of sheaves by $f^{*} T X$. The resulting short exact sequence gives rise a long exact sequence of cohomology groups

$$
\begin{aligned}
0 & \rightarrow B_{2} \rightarrow \bigoplus_{v \in V^{S}(\Gamma)} H^{0}\left(C_{v}\right) \oplus \bigoplus_{e \in E(\Gamma)} H^{0}\left(C_{e}\right) \rightarrow \bigoplus_{v \in V^{2}(\Gamma)} T_{f\left(y_{v}\right)} X \oplus \bigoplus_{(e, v) \in F^{S}(\Gamma)} T_{f(y(e, v))} X \\
& \rightarrow B_{5} \rightarrow \bigoplus_{v \in V^{S}(\Gamma)} H^{1}\left(C_{v}\right) \oplus \bigoplus_{e \in E(\Gamma)} H^{1}\left(C_{e}\right) \rightarrow 0,
\end{aligned}
$$

where

$$
\begin{aligned}
& H^{i}\left(C_{v}\right)=H^{i}\left(C_{v},\left(\left.f\right|_{C_{v}}\right)^{*} T X\right) \cong H^{i}\left(C_{v}, \mathcal{O}_{C_{v}}\right) \otimes T_{p_{\sigma_{v}}} X, \\
& H^{i}\left(C_{e}\right)=H^{i}\left(C_{e},\left(\left.f\right|_{C_{e}}\right)^{*} T X\right)
\end{aligned}
$$

for $i=0,1$. We have

$$
\begin{aligned}
& H^{0}\left(C_{v}\right)=T_{p_{\sigma_{v}}} X, \\
& H^{1}\left(C_{v}\right)=H^{0}\left(C_{v}, \omega_{C_{v}}\right)^{\vee} \otimes T_{p_{\sigma_{v}}} X .
\end{aligned}
$$

Lemma 4.4. Let $\sigma \in V(\Upsilon)$, so that $p_{\sigma}$ is a $T$-fixed point in $X$. Define

$$
\begin{aligned}
& \mathbf{w}(\sigma)=e^{T}\left(T_{p_{\sigma}} X\right) \in H_{T}^{2 r}(\text { point } ; \mathbb{Q}), \\
& \mathbf{h}(\sigma, g)=\frac{e^{T}\left(\mathbb{E}^{\vee} \otimes T_{p_{\sigma}} X\right)}{e^{T}\left(T_{p_{\sigma}} X\right)} \in H_{T}^{2 r(g-1)}\left(\overline{\mathcal{M}}_{g, n} ; \mathbb{Q}\right) .
\end{aligned}
$$

Then

$$
\begin{aligned}
& \mathbf{w}(\sigma)=\prod_{\epsilon \in E_{\sigma}} \mathbf{w}(\epsilon, \sigma), \\
& \mathbf{h}(\sigma, g)=\prod_{\epsilon \in E_{\sigma}} \frac{\Lambda_{g}^{\vee}(\mathbf{w}(\epsilon, \sigma))}{\mathbf{w}(\epsilon, \sigma)},
\end{aligned}
$$

where $\Lambda_{g}^{\vee}(u)=\sum_{i=0}^{g}(-1)^{i} \lambda_{i} u^{g-i}$. 
Proof. $T_{p_{\sigma}} X=\bigoplus_{\epsilon \in E_{\sigma}} T_{p_{\sigma}} \ell_{\epsilon}$, where $e^{T}\left(T_{p_{\sigma}} \ell_{\epsilon}\right)=\mathbf{w}(\epsilon, \sigma)$. So

$$
\begin{aligned}
& e^{T}\left(T_{p_{\sigma}} X\right)=\prod_{\epsilon \in E_{\sigma}} \mathbf{w}(\epsilon, \sigma), \\
& \frac{e^{T}\left(\mathbb{E}^{\vee} \otimes T_{p_{\sigma}} \ell_{\epsilon}\right)}{e^{T}\left(T_{p_{\sigma}} \ell_{\epsilon}\right)}=\prod_{\epsilon \in E_{\sigma}} \frac{e^{T}\left(\mathbb{E}^{\vee} \otimes T_{p_{\sigma}} \ell_{\epsilon}\right)}{\mathbf{w}(\epsilon, \sigma)},
\end{aligned}
$$

where

$$
e^{T}\left(\mathbb{E}^{\vee} \otimes T_{p_{\sigma}} \ell_{\epsilon}\right)=\sum_{i=0}^{g}(-1)^{i} c_{i}(\mathbb{E}) c_{1}^{T}\left(T_{p_{\sigma}} \ell_{\epsilon}\right)^{g-i}=\sum_{i=0}^{g}(-1)^{i} \lambda_{i} \mathbf{w}(\epsilon, \sigma)^{g-i} .
$$

The map $B_{1} \rightarrow B_{2}$ sends

$$
H^{0}\left(C_{e}, T C_{e}\left(-y(e, v)-y\left(e^{\prime}, v\right)\right)\right)
$$

isomorphically to

$$
H^{0}\left(C_{e},\left(\left.f\right|_{C_{e}}\right)^{*} T \ell_{\epsilon_{e}}\right)^{f},
$$

the fixed part of $H^{0}\left(C_{e},\left(\left.f\right|_{C_{e}}\right)^{*} T \ell_{\epsilon_{e}}\right)$.

Lemma 4.5. Given $d \in \mathbb{Z}_{>0}$ and $\epsilon \in E(\Upsilon)_{c}$, define $\sigma, \sigma^{\prime}, \epsilon_{i}, \epsilon_{i}^{\prime}$, a $a_{i}$ as in Section 2.2, and let $f_{d}: \mathbb{P}^{1} \rightarrow \ell_{\epsilon} \cong \mathbb{P}^{1}$ be the unique degree $d$ map totally ramified over the two $T$-fixed points $p_{\sigma}$ and $p_{\sigma^{\prime}}$ in $\ell_{\epsilon}$. Define

$$
\mathbf{h}(\epsilon, d)=\frac{e^{T}\left(H^{1}\left(\mathbb{P}^{1}, f_{d}^{*} T X\right)^{m}\right)}{e^{T}\left(H^{0}\left(\mathbb{P}^{1}, f_{d}^{*} T X\right)^{m}\right)} .
$$

Then

$$
\mathbf{h}(\epsilon, d)=\frac{(-1)^{d} d^{2 d}}{(d !)^{2} \mathbf{w}(\epsilon, \sigma)^{2 d}} \prod_{i=1}^{r-1} b\left(\frac{\mathbf{w}(\epsilon, \sigma)}{d}, \mathbf{w}\left(\epsilon_{i}, \sigma\right), d a_{i}\right),
$$

where

$$
b(u, w, a)= \begin{cases}\prod_{j=0}^{a}(w-j u)^{-1}, & a \in \mathbb{Z}, a \geq 0, \\ \prod_{j=1}^{-a-1}(w+j u), & a \in \mathbb{Z}, a<0 .\end{cases}
$$

Proof. We use the notation in Section 2.2. We have

$$
N_{\ell_{\epsilon} / X}=L_{1} \oplus \cdots \oplus L_{r-1} .
$$

The weights of $T$-actions on $\left(L_{i}\right)_{p_{\sigma}}$ and $\left(L_{i}\right)_{p_{\sigma}}$ are $\mathbf{w}\left(\epsilon_{i}, \sigma\right)$ and $\mathbf{w}\left(\epsilon_{i}, \sigma\right)-a_{i} \mathbf{w}(\epsilon, \sigma)$, respectively. The weights of $T$-actions on $T_{0} \mathbb{P}^{1}, T_{\infty} \mathbb{P}^{1},\left(f_{d}^{*} L_{i}\right)_{0},\left(f_{d}^{*} L_{i}\right)_{\infty}$ are $u:=\frac{\mathbf{w}(\epsilon, \sigma)}{d},-u, w_{i}:=\mathbf{w}\left(\epsilon_{i}, \sigma\right)$, $w_{i}-d a_{i} u$, respectively. By [23, Example 19],

$$
\operatorname{ch}_{T}\left(H^{0}\left(\mathbb{P}^{1}, f_{d}^{*} L_{i}\right)-H^{1}\left(\mathbb{P}^{1}, f_{d}^{*} L_{i}\right)\right)= \begin{cases}\sum_{j=0}^{d a_{i}} e^{w_{i}-j u}, & a_{i} \geq 0, \\ \sum_{j=1}^{-d a_{i}-1} e^{w_{i}+j u}, & a_{i}<0 .\end{cases}
$$


Note that $w_{i}+j u$ is nonzero for any $j \in \mathbb{Z}$ since $w_{i}$ and $u$ are linearly independent for $i=1, \ldots, n-1$. So

$$
\frac{e^{T}\left(H^{1}\left(\mathbb{P}^{1}, f_{d}^{*} L_{i}\right)\right)}{e^{T}\left(H^{0}\left(\mathbb{P}^{1}, f_{d}^{*} L_{i}\right)\right)}=\frac{e^{T}\left(H^{1}\left(\mathbb{P}^{1}, f_{d}^{*} L_{i}\right)^{m}\right)}{e^{T}\left(H^{0}\left(\mathbb{P}^{1}, f_{d}^{*} L_{i}\right)^{m}\right)}=b\left(u, w_{i}, d a_{i}\right),
$$

where $b(u, w, a)$ is defined by (4.8). By [23, Example 19],

$$
\operatorname{ch}_{T}\left(H^{0}\left(\mathbb{P}^{1}, f_{d}^{*} T \ell_{\epsilon}\right)-H^{1}\left(\mathbb{P}^{1}, f_{d}^{*} T \ell_{\epsilon}\right)\right)=\sum_{j=0}^{2 d} e^{d u-j u}=1+\sum_{j=1}^{d}\left(e^{j \mathbf{w}(\epsilon, \sigma) / d}+e^{-j \mathbf{w}(\epsilon, \sigma) / d}\right) .
$$

So

$$
\frac{e^{T}\left(H^{1}\left(\mathbb{P}^{1}, f_{d}^{*} T \ell_{\epsilon}\right)^{m}\right)}{e^{T}\left(H^{0}\left(\mathbb{P}^{1}, f_{d}^{*} T \ell_{\epsilon}\right)^{m}\right)}=\prod_{j=1}^{d} \frac{-d^{2}}{j^{2} \mathbf{w}(\epsilon, \sigma)^{2}}=\frac{(-1)^{d} d^{2 d}}{(d !)^{2} \mathbf{w}(\epsilon, \sigma)^{2 d}}
$$

Therefore,

$$
\begin{aligned}
\frac{e^{T}\left(H^{1}\left(\mathbb{P}^{1}, f_{d}^{*} T X\right)^{m}\right)}{e^{T}\left(H^{0}\left(\mathbb{P}^{1}, f_{d}^{*} T X\right)^{m}\right)} & =\frac{e^{T}\left(H^{1}\left(\mathbb{P}^{1}, f_{d}^{*} T \ell_{\epsilon}\right)^{m}\right)}{e^{T}\left(H^{0}\left(\mathbb{P}^{1}, f_{d}^{*} T \ell_{\epsilon}\right)^{m}\right)} \prod_{i=1}^{r-1} \frac{e^{T}\left(H^{1}\left(\mathbb{P}^{1}, f_{d}^{*} L_{i}\right)^{m}\right)}{e^{T}\left(H^{0}\left(\mathbb{P}^{1}, f_{d}^{*} L_{i}\right)^{m}\right)} \\
& =\frac{(-1)^{d} d^{2 d}}{(d !)^{2} \mathbf{w}(\epsilon, \sigma)^{2 d}} \prod_{i=1}^{r-1} b\left(\frac{\mathbf{w}(\epsilon, \sigma)}{d}, \mathbf{w}\left(\epsilon_{i}, \sigma\right), d a_{i}\right) .
\end{aligned}
$$

Finally, $f\left(y_{v}\right)=p_{\sigma_{v}}=f(y(e, v))$, and

$$
e^{T}\left(T_{p_{\sigma_{v}}} X\right)=\mathbf{w}\left(\sigma_{v}\right) .
$$

From the above discussion, we conclude that

$$
\begin{aligned}
\frac{e^{T}\left(B_{5}^{m}\right)}{e^{T}\left(B_{2}^{m}\right)} & =\prod_{v \in V^{2}(\Gamma)} \mathbf{w}\left(\sigma_{v}\right) \prod_{(e, v) \in F^{S}(\Gamma)} \mathbf{w}\left(\sigma_{v}\right) \prod_{v \in V^{S}(\Gamma)} \mathbf{h}\left(\sigma_{v}, g_{v}\right) \prod_{e \in E(\Gamma)} \mathbf{h}\left(\epsilon_{e}, d_{e}\right) \\
& =\prod_{v \in V(\Gamma)}\left(\mathbf{h}\left(\sigma_{v}, g_{v}\right) \mathbf{w}\left(\sigma_{v}\right)^{\operatorname{val}(v)}\right) \prod_{e \in E(\Gamma)} \mathbf{h}\left(\epsilon_{e}, d_{e}\right)
\end{aligned}
$$

where $\mathbf{w}(\sigma), \mathbf{h}(\sigma, g)$, and $\mathbf{h}(\epsilon, d)$ are defined by (4.5), (4.6), (4.7), respectively.

\subsection{Contribution from each graph}

\subsubsection{Virtual tangent bundle}

We have $B_{1}^{f}=B_{2}^{f}, B_{5}^{f}=0$. So

$$
T^{1, f}=B_{4}^{f}=\bigoplus_{v \in V^{S}(\Gamma)} T_{\left(C_{v}, \mathbf{x}_{v}\right)} \overline{\mathcal{M}}_{g_{v}, E_{v} \cup S_{v}}, \quad T^{2, f}=0 .
$$

We conclude that

$$
\left[\prod_{v \in V^{S}(\Gamma)} \overline{\mathcal{M}}_{g_{v}, E_{v} \cup S_{v}}\right]^{\mathrm{vir}}=\prod_{v \in V^{S}(\Gamma)}\left[\overline{\mathcal{M}}_{g_{v}, E_{v} \cup S_{v}}\right] .
$$




\subsubsection{Virtual normal bundle}

Let $N_{\vec{\Gamma}}^{\text {vir }}$ be the pull back of the virtual normal bundle of $\mathcal{F}_{\vec{\Gamma}}$ in $\overline{\mathcal{M}}_{g, n}(X, \beta)$ under $i_{\vec{\Gamma}}: \mathcal{M}_{\vec{\Gamma}} \rightarrow \mathcal{F}_{\vec{\Gamma}}$. Then

$$
\frac{1}{e^{T}\left(N_{\vec{\Gamma}}^{\mathrm{vir}}\right)}=\frac{e^{T}\left(B_{1}^{m}\right) e^{T}\left(B_{5}^{m}\right)}{e^{T}\left(B_{2}^{m}\right) e^{T}\left(B_{4}^{m}\right)}=\prod_{v \in V(\Gamma)} \frac{\mathbf{h}\left(\sigma_{v}, g_{v}\right) \mathbf{w}\left(\sigma_{v}\right)^{\operatorname{val}(v)}}{\prod_{e \in E_{v}}\left(w_{(e, v)}-\psi_{(e, v)}\right)} \prod_{e \in E(\Gamma)} \mathbf{h}\left(\epsilon_{e}, d_{e}\right) .
$$

\subsubsection{Integrand}

Given $\sigma \in V(\Upsilon)$, let

$$
i_{\sigma}^{*}: A_{T}^{*}(X) \rightarrow A_{T}^{*}\left(p_{\sigma}\right)=\mathbb{Q}\left[u_{1}, \ldots, u_{r}\right]
$$

be induced by the inclusion $i_{\sigma}: p_{\sigma} \rightarrow X$. Then

$$
\begin{aligned}
i_{\vec{\Gamma}}^{*} \prod_{i=1}^{n}\left(\mathrm{ev}_{i}^{*} \gamma_{i}^{T} \cup\left(\psi_{i}^{T}\right)^{a_{i}}\right) & \\
& =\prod_{\substack{v \in V^{1,1}(E) \\
S_{v}=\{i\}, E_{v}=\{e\}}} i_{\sigma_{v}}^{*} \gamma_{i}^{T}\left(-w_{(e, v)}\right)^{a_{i}} \prod_{v \in V^{S}(\Gamma)}\left(\prod_{i \in S_{v}} i_{\sigma_{v}}^{*} \gamma_{i}^{T} \prod_{e \in E_{v}} \psi_{(e, v)}^{a_{i}}\right) .
\end{aligned}
$$

To unify the stable vertices in $V^{S}(\Gamma)$ and the unstable vertices in $V^{1,1}(\Gamma)$, we use the following convention: for $a \in \mathbb{Z}_{\geq 0}$,

$$
\int_{\overline{\mathcal{M}}_{0,2}} \frac{\psi_{2}^{a}}{w_{1}-\psi_{1}}=\left(-w_{1}\right)^{a}
$$

In particular, (4.4) is obtained by setting $a=0$. With the convention (4.10), we may rewrite (4.9) as

$$
i_{\vec{\Gamma}}^{*} \prod_{i=1}^{n}\left(\operatorname{ev}_{i}^{*} \gamma_{i}^{T} \cup\left(\psi_{i}^{T}\right)^{a_{i}}\right)=\prod_{v \in V(\Gamma)}\left(\prod_{i \in S_{v}} i_{\sigma_{v}}^{*} \gamma_{i}^{T} \prod_{e \in E_{v}} \psi_{(e, v)}^{a_{i}}\right)
$$

The following lemma shows that the convention (4.10) is consistent with the stable case $\overline{\mathcal{M}}_{0, n}$, $n \geq 3$.

Lemma 4.6. Let $n$, $a$ be integers, $n \geq 2, a \geq 0$. Then

$$
\int_{\overline{\mathcal{M}}_{0, n}} \frac{\psi_{2}^{a}}{w_{1}-\psi_{1}}= \begin{cases}\frac{\prod_{i=0}^{a-1}(n-3-i)}{a !} w_{1}^{a+2-n}, & n=2 \text { or } 0 \leq a \leq n-3 \\ 0, & \text { otherwise. }\end{cases}
$$

Proof. The case $n=2$ follows from (4.10). For $n \geq 3$,

$$
\begin{aligned}
\int_{\overline{\mathcal{M}}_{0, n}} \frac{\psi_{2}^{a}}{w_{1}-\psi_{1}} & =\frac{1}{w_{1}} \int_{\overline{\mathcal{M}}_{0, n}} \frac{\psi_{2}^{a}}{1-\frac{\psi_{1}}{w_{1}}}=w_{1}^{a+2-n} \int_{\overline{\mathcal{M}}_{0, n}} \psi_{1}^{n-3-a} \psi_{2}^{a} \\
& =w_{1}^{a+2-n} \frac{(n-3) !}{(n-3-a) ! a_{!}}=\frac{\prod_{i=0}^{a-1}(n-3-i)}{a !} w_{1}^{a+2-n} .
\end{aligned}
$$




\subsubsection{Integral}

The contribution of

$$
\int_{\left[\overline{\mathcal{M}}_{g, n}(X, \beta)^{T}\right]^{\mathrm{vir}, T}} \frac{i^{*} \prod_{i=1}^{n}\left(\mathrm{ev}_{i}^{*} \gamma_{i}^{T} \cup\left(\psi_{i}^{T}\right)^{a_{i}}\right)}{e^{T}\left(N^{\mathrm{vir}}\right)}
$$

from the fixed locus $\mathcal{F}_{\vec{\Gamma}}$ is given by

$$
\frac{1}{\left|A_{\vec{\Gamma}}\right|} \prod_{e \in E(\Gamma)} \mathbf{h}\left(\epsilon_{e}, d_{e}\right) \prod_{v \in V(\Gamma)}\left(\mathbf{w}\left(\sigma_{v}\right)^{\operatorname{val}(v)} \prod_{i \in S_{v}} i_{\sigma_{v}}^{*} \gamma_{i}^{T}\right) \prod_{v \in V(\Gamma)} \int_{\overline{\mathcal{M}}_{g_{v}, E_{v} \cup S_{v}}} \frac{\mathbf{h}\left(\sigma_{v}, g_{v}\right) \prod_{e \in E_{v}} \psi_{(e, v)}^{a_{i}}}{\prod_{e \in E_{v}}\left(w_{(e, v)}-\psi_{(e, v)}\right)},
$$

where $\left|A_{\vec{\Gamma}}\right|=|\operatorname{Aut}(\vec{\Gamma})| \prod_{e \in E(\Gamma)} d_{e}$.

\subsection{Sum over graphs}

Summing over the contribution from each graph $\vec{\Gamma}$ given in Section 4.3 .4 above, we obtain the following formula.

\section{Theorem 4.7.}

$$
\begin{aligned}
& \left\langle\tau_{a_{1}}\left(\gamma_{1}^{T}\right) \cdots \tau_{a_{n}}\left(\gamma_{n}^{T}\right)\right\rangle_{g, \beta}^{X_{T}} \\
& =\sum_{\vec{\Gamma} \in G_{g, n}(X, \beta)} \frac{1}{|\operatorname{Aut}(\vec{\Gamma})|} \prod_{e \in E(\Gamma)} \frac{\mathbf{h}\left(\epsilon_{e}, d_{e}\right)}{d_{e}} \prod_{v \in V(\Gamma)}\left(\mathbf{w}\left(\sigma_{v}\right)^{\operatorname{val}(v)} \prod_{i \in S_{v}} i_{\sigma_{v}}^{*} \gamma_{i}^{T}\right) \\
& \quad \times \prod_{v \in V(\Gamma)} \int_{\overline{\mathcal{M}}_{g, E v} \cup S_{v}} \frac{\mathbf{h}\left(\sigma_{v}, g_{v}\right) \prod_{i \in S_{v}} \psi_{i}^{a_{i}}}{\prod_{e \in E_{v}}\left(w_{(e, v)}-\psi_{(e, v)}\right)},
\end{aligned}
$$

where $\mathbf{h}(\epsilon, d), \mathbf{w}(\sigma), \mathbf{h}(\sigma, g)$ are given by (4.7), (4.5), (4.6), respectively, and we have the following convention for the $v \notin V^{S}(\Gamma)$ :

$$
\begin{aligned}
\int_{\overline{\mathcal{M}}_{0,1}} \frac{1}{w_{1}-\psi_{2}} & =w_{1}, \quad \int_{\overline{\mathcal{M}}_{0,2}} \frac{1}{\left(w_{1}-\psi_{1}\right)\left(w_{2}-\psi_{2}\right)}=\frac{1}{w_{1}+w_{2}}, \\
\int_{\overline{\mathcal{M}}_{0,2}} \frac{\psi_{2}^{a}}{w_{1}-\psi_{1}} & =\left(-w_{1}\right)^{a}, \quad a \in \mathbb{Z}_{\geq 0} .
\end{aligned}
$$

Given $g \in \mathbb{Z}_{\geq 0}, r$ weights $\vec{w}=\left\{w_{1}, \ldots, w_{r}\right\}, r$ partitions $\vec{\mu}=\left\{\mu^{1}, \ldots, \mu^{r}\right\}$, and $a_{1}, \ldots, a_{k} \in \mathbb{Z}$, let $\ell\left(\mu^{i}\right)$ be the length of $\mu^{i}$, and let $\ell(\vec{\mu})=\sum_{i=1}^{r} \ell\left(\mu^{i}\right)$. We define

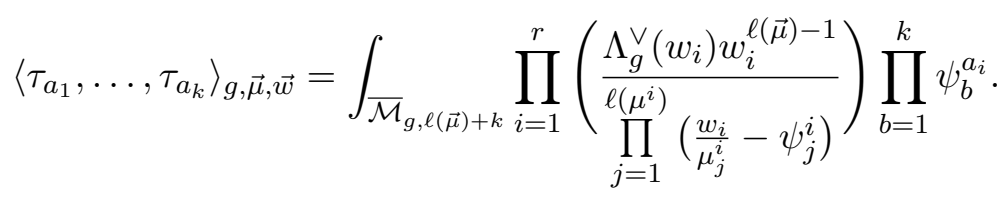

Given $v \in V(\Gamma)$, define $\vec{w}(v)=\left\{\mathbf{w}\left(\epsilon, \sigma_{v}\right) \mid\left(\epsilon, \sigma_{v}\right) \in F(\Upsilon)\right\}$. Given $v \in V(\Gamma)$, and $\epsilon \in E_{\sigma_{v}}$, let $\mu^{v, \epsilon}$ be a (possibly empty) partition defined by $\left\{d_{e} \mid e \in E_{v}, \vec{f}(e)=\epsilon\right\}$, and define $\vec{\mu}(v)=$ $\left\{\mu^{v, \epsilon} \mid\left(\epsilon, \sigma_{v}\right) \in F(\Upsilon)\right\}$. Then (4.11) can be rewritten as

$$
\left\langle\tau_{a_{1}}\left(\gamma_{1}^{T}\right) \cdots \tau_{a_{n}}\left(\gamma_{n}^{T}\right)\right\rangle_{g, \beta}^{X_{T}}
$$




$$
=\sum_{\vec{\Gamma} \in G_{g, n}(X, \beta)} \frac{1}{|\operatorname{Aut}(\vec{\Gamma})|} \prod_{e \in E(\Gamma)} \frac{\mathbf{h}\left(\epsilon_{e}, d_{e}\right)}{d_{e}} \prod_{v \in V(\Gamma)}\left(\prod_{i \in S_{v}} i_{\sigma_{v}}^{*} \gamma_{i}\left\langle\prod_{i \in S_{v}} \tau_{a_{i}}\right\rangle_{g_{v}, \vec{\mu}(v), \vec{w}(v)}\right) .
$$

Recall that

$$
g=\sum_{v \in V(\Gamma)} g_{v}+|E(\Gamma)|-|V(\Gamma)|+1
$$

SO

$$
2 g-2=\sum_{v \in V(\Gamma)}\left(2 g_{v}-2+\operatorname{val}(v)\right)
$$

Given $\vec{\Gamma}=(\Gamma, \vec{f}, \vec{d}, \vec{g}, \vec{s})$, let $\vec{\Gamma}^{\prime}=(\Gamma, \vec{f}, \vec{d}, \vec{s})$ be the decorated graph obtained by forgetting the genus map. Let $G_{n}(X, \beta)=\left\{\vec{\Gamma}^{\prime} \mid \vec{\Gamma} \in \cup_{g \geq 0} G_{g, n}(X, \beta)\right\}$. Define

$$
\begin{aligned}
& \left\langle\tau_{a_{1}}\left(\gamma_{1}^{T}\right), \ldots, \tau_{a_{n}}\left(\gamma_{n}^{T}\right) \mid u\right\rangle_{\beta}^{X_{T}}=\sum_{g \geq 0} u^{2 g-2}\left\langle\tau_{a_{1}}\left(\gamma_{1}^{T}\right), \ldots, \tau_{a_{n}}\left(\gamma_{n}^{T}\right)\right\rangle_{g, \beta}^{X_{T}}, \\
& \left\langle\tau_{a_{1}}, \ldots, \tau_{a_{k}} \mid u\right\rangle_{\vec{\mu}, \vec{w}}=\sum_{g \geq 0} u^{2 g-2+\ell(\vec{\mu})}\left\langle\tau_{a_{1}}, \ldots, \tau_{a_{k}}\right\rangle_{g, \vec{\mu}, \vec{w}} .
\end{aligned}
$$

Then we have the following formula for the generating function (4.12).

\section{Theorem 4.8.}

$$
\begin{aligned}
\left\langle\tau_{a_{1}}\left(\gamma_{1}^{T}\right) \cdots \tau_{a_{n}}\left(\gamma_{n}^{T}\right) \mid u\right\rangle_{\beta}^{X_{T}}= & \sum_{\vec{\Gamma}^{\prime} \in G_{n}(X, \beta)} \frac{1}{|\operatorname{Aut}(\vec{\Gamma})|} \prod_{e \in E(\Gamma)} \frac{\mathbf{h}\left(\epsilon_{e}, d_{e}\right)}{d_{e}} \\
& \times \prod_{v \in V(\Gamma)}\left(\prod_{i \in S_{v}} i_{\sigma_{v}}^{*} \gamma_{i}^{T}\left\langle\prod_{i \in S_{v}} \tau_{a_{i}} \mid u\right\rangle_{\vec{\mu}(v), \vec{w}(v)}\right) .
\end{aligned}
$$

\section{Acknowledgements}

The first author would like to thank Tom Graber for his suggestion of generalizing the computations for toric manifolds in [23] to GKM manifolds. The second author would like to thank the Columbia University for hospitality during his visits. We wish to thank Rahul Pandharipande for his comments on an earlier version of this paper. This work is partially supported by NSF DMS-1159416 and NSF DMS-1206667.

\section{References}

[1] Atiyah M.F., Bott R., The moment map and equivariant cohomology, Topology 23 (1984), 1-28.

[2] Behrend K., Gromov-Witten invariants in algebraic geometry, Invent. Math. 127 (1997), 601-617, alg-geom/9601011.

[3] Behrend K., Localization and Gromov-Witten invariants, in Quantum Cohomology (Cetraro, 1997), Lecture Notes in Math., Vol. 1776, Springer, Berlin, 2002, 3-38.

[4] Behrend K., Fantechi B., The intrinsic normal cone, Invent. Math. 128 (1997), 45-88, alg-geom/9601010.

[5] Behrend K., Manin Yu., Stacks of stable maps and Gromov-Witten invariants, Duke Math. J. 85 (1996), 1-60, alg-geom/9506023.

[6] Chen L., Li Y., Liu K., Localization, Hurwitz numbers and the Witten conjecture, Asian J. Math. 12 (2008), 511-518, math.AG/0609263.

[7] Deligne P., Mumford D., The irreducibility of the space of curves of given genus, Inst. Hautes Études Sci. Publ. Math. 36 (1969), 75-109. 
[8] Faber C., Algorithms for computing intersection numbers on moduli spaces of curves, with an application to the class of the locus of Jacobians, in New Trends in Algebraic Geometry (Warwick, 1996), London Math. Soc. Lecture Note Ser., Vol. 264, Cambridge University Press, Cambridge, 1999, 93-109, alg-geom/9706006.

[9] Goresky M., Kottwitz R., MacPherson R., Equivariant cohomology, Koszul duality, and the localization theorem, Invent. Math. 131 (1998), 25-83.

[10] Graber T., Pandharipande R., Localization of virtual classes, Invent. Math. 135 (1999), 487-518, alg-geom/9708001.

[11] Guillemin V., Zara C., Equivariant de Rham theory and graphs, Asian J. Math. 3 (1999), 49-76, math.AG/9808135.

[12] Hartshorne R., Algebraic geometry, Graduate Texts in Mathematics, Vol. 52, Springer-Verlag, New York Heidelberg, 1977.

[13] Hori K., Katz S., Klemm A., Pandharipande R., Thomas R., Vafa C., Vakil R., Zaslow E., Mirror symmetry, Clay Mathematics Monographs, Vol. 1, Amer. Math. Soc., Providence, RI, Clay Mathematics Institute, Cambridge, MA, 2003.

[14] Kazarian M., KP hierarchy for Hodge integrals, Adv. Math. 221 (2009), 1-21, arXiv:0809.3263.

[15] Kazarian M.E., Lando S.K., An algebro-geometric proof of Witten's conjecture, J. Amer. Math. Soc. 20 (2007), 1079-1089, math.AG/0601760.

[16] Kim Y.-S., Liu K., Virasoro constraints and Hurwitz numbers through asymptotic analysis, Pacific J. Math. 241 (2009), 275-284.

[17] Knudsen F.F., Mumford D., The projectivity of the moduli space of stable curves. I. Preliminaries on "det" and "Div", Math. Scand. 39 (1976), 19-55.

[18] Knudsen F.F., The projectivity of the moduli space of stable curves. II. The stacks $M_{g, n}$, Math. Scand. 52 (1983), 161-199.

[19] Knudsen F.F., The projectivity of the moduli space of stable curves. III. The line bundles on $M_{g, n}$, and a proof of the projectivity of $\bar{M}_{g, n}$ in characteristic 0, Math. Scand. 52 (1983), 200-212.

[20] Kontsevich M., Intersection theory on the moduli space of curves and the matrix Airy function, Comm. Math. Phys. 147 (1992), 1-23.

[21] Kontsevich M., Enumeration of rational curves via torus actions, in The Moduli Space of Curves (Texel Island, 1994), Progr. Math., Vol. 129, Birkhäuser Boston, Boston, MA, 1995, 335-368, hep-th/9405035.

[22] Li J., Tian G., Virtual moduli cycles and Gromov-Witten invariants of algebraic varieties, J. Amer. Math. Soc. 11 (1998), 119-174, alg-geom/9602007.

[23] Liu C.-C.M., Localization in Gromov-Witten theory and orbifold Gromov-Witten theory, in Handbook of Moduli, Vol. II, Adv. Lect. Math. (ALM), Vol. 25, Int. Press, Somerville, MA, 2013, 353-425, arXiv:1107.4712.

[24] Mirzakhani M., Weil-Petersson volumes and intersection theory on the moduli space of curves, J. Amer. Math. Soc. 20 (2007), 1-23.

[25] Mulase M., Zhang N., Polynomial recursion formula for linear Hodge integrals, Commun. Number Theory Phys. 4 (2010), 267-293, arXiv:0908.2267.

[26] Mumford D., Towards an enumerative geometry of the moduli space of curves, in Arithmetic and Geometry, Vol. II, Progr. Math., Vol. 36, Birkhäuser Boston, Boston, MA, 1983, 271-328.

[27] Okounkov A., Pandharipande R., Gromov-Witten theory, Hurwitz numbers, and matrix models, in Algebraic Geometry - Seattle 2005, Part 1, Proc. Sympos. Pure Math., Vol. 80, Amer. Math. Soc., Providence, RI, 2009, 325-414, math.AG/0101147.

[28] Spielberg H., A formula for the Gromov-Witten invariants of toric varieties, Ph.D. Thesis, Université Louis Pasteur (Strasbourg I), Strasbourg, 1999, math.AG/0006156.

[29] Witten E., Two-dimensional gravity and intersection theory on moduli space, in Surveys in Differential Geometry (Cambridge, MA, 1990), Lehigh University, Bethlehem, PA, 1991, 243-310. 